Purdue University Purdue e-Pubs

Open Access Theses

Theses and Dissertations

$12-2016$

\title{
A small-scale testbed for large-scale reliable computing
}

Jason R. St. John

Purdue University

Follow this and additional works at: https://docs.lib.purdue.edu/open_access_theses

Part of the Computer Sciences Commons

\section{Recommended Citation}

St. John, Jason R., "A small-scale testbed for large-scale reliable computing" (2016). Open Access Theses. 899. https://docs.lib.purdue.edu/open_access_theses/899

This document has been made available through Purdue e-Pubs, a service of the Purdue University Libraries. Please contact epubs@purdue.edu for additional information. 


\section{PURDUE UNIVERSITY \\ GRADUATE SCHOOL \\ Thesis/Dissertation Acceptance}

This is to certify that the thesis/dissertation prepared

By Jason R. St. John

Entitled

A SMALL-SCALE TESTBED FOR LARGE-SCALE RELIABLE COMPUTING

For the degree of Master of Science

Is approved by the final examining committee:

Thomas Hacker

Chair

Eric Matson

John Springer

To the best of my knowledge and as understood by the student in the Thesis/Dissertation Agreement, Publication Delay, and Certification Disclaimer (Graduate School Form 32), this thesis/dissertation adheres to the provisions of Purdue University's "Policy of Integrity in Research” and the use of copyright material.

Approved by Major Professor(s): Thomas Hacker

Approved by:

Jeffrey Whitten

$11 / 29 / 2016$

Head of the Departmental Graduate Program

Date 



\title{
A SMALL-SCALE TESTBED FOR
}

\section{LARGE-SCALE RELIABLE COMPUTING}

\author{
A Thesis \\ Submitted to the Faculty \\ of \\ Purdue University \\ by \\ Jason R. St. John \\ In Partial Fulfillment of the
Requirements for the Degree \\ of \\ Master of Science
}

December 2016

Purdue University

West Lafayette, Indiana 


\section{ACKNOWLEDGMENTS}

I wish to gratefully acknowledge my thesis committee, my family, and my fellow graduate students for their help and support, encouragement, and insight. 
TABLE OF CONTENTS

Page

LIST OF TABLES . . . . . . . . . . . . . . . . . . . . . v

LIST OF FIGURES . . . . . . . . . . . . . . . . . . . vi

ABBREVIATIONS ................................. vii

ABSTRACT ............................. ix

CHAPTER 1. INTRODUCTION . . . . . . . . . . . . . . . . . . . . . 1

1.1 Scope . . . . . . . . . . . . . . . . . . . . . . 1

1.2 Significance . . . . . . . . . . . . . . . . . . . . . 1

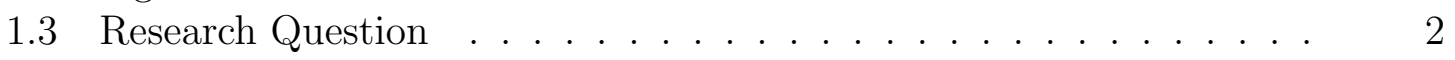

1.4 Assumptions . . . . . . . . . . . . . . . . . . . 2

1.5 Limitations . . . . . . . . . . . . . . . . . . . 3

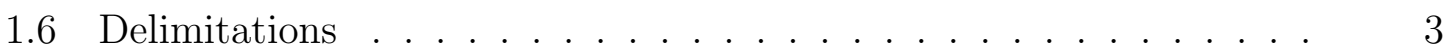

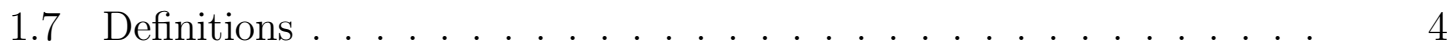

1.8 Summary . . . . . . . . . . . . . . . . . . . 5

CHAPTER 2. REVIEW OF RELEVANT LITERATURE . . . . . . . . . . 6

2.1 HPC System Reliability . . . . . . . . . . . . . . . . 6

2.2 HPC, Cloud Computing, and Virtualization . . . . . . . . . . 8

2.3 Error Injection . . . . . . . . . . . . . . . . . . . . . 9

2.4 Summary . . . . . . . . . . . . . . . . . . . 11

CHAPTER 3. FRAMEWORK AND METHODOLOGY . . . . . . . . . . . 12

3.1 Study Design . . . . . . . . . . . . . . . . . . . . . . . . . 12

3.1 .1 Data Sets . . . . . . . . . . . . . . . . . 12

3.1.2 Overview of Statistical Model Creation . . . . . . . . . . . 13

3.1.3 System Log Analysis . . . . . . . . . . . . . . . . . . . 14

3.1.3.1 Coates: Log Processing and Sorting . . . . . . . . . 14

3.1.3.2 Coates: Timestamp Standardization . . . . . . . . 15

3.1.3.3 Coates: Node ID Standardization . . . . . . . . . . 15

3.1.3.4 Coates: Scatter Plots of Memory Failure Events . . 16

3.1.3.5 Coates: Declustering of Failure Events . . . . . . . 16

3.1.3.6 Carter: Scatter Plots of Hard Disk Failure Events . 16

3.1.3.7 Carter: Declustering of Failure Events . . . . . . . 16

3.1.3.8 Distribution Fitting . . . . . . . . . . . . 21

3.1.4 Fault Injection . . . . . . . . . . . . . . . . 21 
3.1.4.1 Memory Error Injection Procedure . . . . . . . . . 22

3.1.4.2 Machine Check Registers . . . . . . . . . . . 22

3.1.4.3 Memory Error Injection Strings . . . . . . . . . . . 23

3.1.4.4 QEMU Modifications and Hard Disk Error Simulation Procedure ................ . . 23

3.1.4.5 Hard Disk Errors . . . . . . . . . . . . . . . 24

3.1.4.6 Tunable Fault Injection Frequency . . . . . . . . . 25

3.1.4.7 Tuning the Three-Parameter Weibull Distribution . 25

3.1.4.8 Tuning the Lomax Distribution . . . . . . . . . . 25

3.1.4.9 Experimental Setup . . . . . . . . . . 26

3.2 Unit \& Sampling . . . . . . . . . . . . . . . . . . . . . . 27

3.2.1 Hypothesis. . . . . . . . . . . . . . . . . . . . 27

3.2.2 Population . . . . . . . . . . . . . . . . . . . . . 27

3.2 .3 Sample . . . . . . . . . . . . . . . . . 31

3.2 .4 Variables . . . . . . . . . . . . . . . . . . . . . . 31

3.2.5 Measure for Success . . . . . . . . . . . . . . . . . . . . . 31

3.3 Summary . . . . . . . . . . . . . . . . . . . . 31

CHAPTER 4. PRESENTATION OF DATA . . . . . . . . . . . . . . . . . . 32

4.1 Statistical Models . . . . . . . . . . . . . . . . 32

4.1.1 Coates: Memory Errors . . . . . . . . . . . . . . . . 32

4.1.2 Carter: Hard Disk Errors . . . . . . . . . . . . . . . . 33

4.2 Experiments . . . . . . . . . . . . . . . . . . . . . . . . . . . . . . . . . . .

4.2.1 System Log Samples . . . . . . . . . . . . . . . . . . . . 33

4.2.2 Quantitative Results ............... 33

CHAPTER 5. CONCLUSIONS, DISCUSSION, AND RECOMMENDATIONS 36

5.1 Conclusions and Discussion . . . . . . . . . . . . 36

5.2 Recommendations . . . . . . . . . . . . . . . 37

APPENDIX A. FAULT INJECTION SCRIPT . . . . . . . . . . . . . . . 38

APPENDIX B. QEMU SOURCE CODE MODIFICATIONS . . . . . . . . . 51

LIST OF REFERENCES . . . . . . . . . . . . . . . . . 65 


\section{LIST OF TABLES}

Table $\quad$ Page

3.1 Comparison of various timestamp formats . . . . . . . . . . . . 15

3.2 Polynomial coefficients . . . . . . . . . . . . . . . . . . . 19

3.3 Polynomial roots . . . . . . . . . . . . . . . . . . 20

3.4 Number of centers vs. WSSE . . . . . . . . . . . . . . . . . . 21

3.5 Supported memory errors and $M C i_{-} S T A T U S$ register values . . . . . . 23

3.6 Supported hard disk errors and their descriptions . . . . . . . . . . . . 24

4.1 Parameters of the three-parameter Weibull distribution for integrated memory controller errors on the Coates system . . . . . . . . . . . . . . . 32

4.2 Parameters of the Lomax distribution for hard disk errors on the Carter

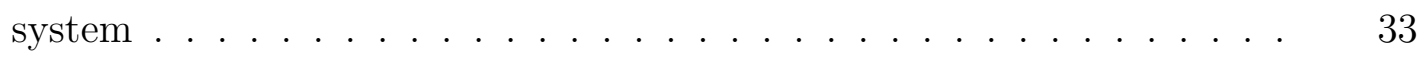

4.3 Number of attempted and successful fault injections . . . . . . . . . . . 35 


\section{LIST OF FIGURES}

Figure $\quad$ Page

3.1 Time of Memory Error Events vs. Node ID . . . . . . . . . . . . . . 17

3.2 Time of Hard Disk Error Events vs. Node ID . . . . . . . . . . . . . . 18

3.3 WSSE vs. Number of Clusters . . . . . . . . . . . . . . . . . 20

3.4 Configuration file for fio . . . . . . . . . . . . . . . . . . 28

3.5 systemd service file for fio, installed on the VMs . . . . . . . . . . . . . 29

3.6 systemd service file for changing the default polling interval for machine checks . . . . . . . . . . . . . . . . . 29

3.7 Bash script that changes the default polling frequency for machine checks 30

4.1 System log screenshot of VM "centos4" experiencing an ENOMEDIUM error injection . . . . . . . . . . . . . . . . . 


\title{
ABBREVIATIONS
}

\author{
ADDR memory address \\ BSD Berkeley Software Distribution \\ CPU central processing unit \\ EC2 Amazon's Elastic Compute Cloud \\ ECC error-correcting code \\ DRAM dynamic random-access memory \\ fio Flexible I/O Tester \\ GART Graphics Address Relocation Table \\ GUI graphical user interface \\ HMP QEMU's Human Monitor Protocol \\ HPC high performance computing \\ IaaS infrastructure as a service \\ I/O input/output \\ IP Internet Protocol \\ ISO International Organization for Standardization \\ IT information technology \\ MAC media access control \\ MC machine check \\ MCA machine check architecture \\ MCE machine check exception \\ MTBF mean time between failures \\ MTTF mean time to failure \\ RAM random-access memory \\ RCAC Rosen Center for Advanced Computing
}


RFC Request For Comments

RHEL Red Hat Enterprise Linux

TLB Translation Lookaside Buffer

UTC Coordinated Universal Time

VM virtual machine

VMM virtual machine monitor

WSSE within groups sum of squared error 


\begin{abstract}
St. John, Jason R. M.S., Purdue University, December 2016. A Small-Scale Testbed For Large-Scale Reliable Computing. Major Professor: Thomas J. Hacker.

High performance computing (HPC) systems frequently suffer errors and failures from hardware components that negatively impact the performance of jobs run on these systems. We analyzed system logs from two HPC systems at Purdue University and created statistical models for memory and hard disk errors. We created a small-scale error injection testbed - using a customized QEMU build, libvirt, and Python - for HPC application programmers to test and debug their programs in a faulty environment so that programmers can write more robust and resilient programs before deploying them on an actual HPC system. The deliverables for this project are the fault injection program, the modified QEMU source code, and the statistical models used for driving the injection.
\end{abstract}




\section{CHAPTER 1. INTRODUCTION}

High performance computing (HPC) systems are a major component of academic and industrial scientific research, and the reliability of these systems is one of the foremost areas of research in the HPC community. The HPC community is continually trying to make larger, more powerful HPC systems, and the poor reliability of commodity HPC clusters is one of the biggest hindrances to the adoption of petascale and exascale computing systems. This chapter provides the scope, significance, research question, and other background information on the thesis.

\subsection{Scope}

This study examined the system logs of large-scale HPC systems operated by the Rosen Center for Advanced Computing (RCAC) at Purdue University. The systems studied are all commodity-based computing clusters running Red Hat Enterprise Linux (RHEL) 5, which is a GNU/Linux distribution based on version 2.6.18 of the Linux kernel. This study determined the frequency at which component failures occurred and produced statistical models of the recorded failure events. The statistical models were used as the driving input to a synthetic fault generator that simulated hardware failures within a virtual machine environment.

\section{$\underline{1.2 \text { Significance }}$}

Large-scale high performance computing (HPC) systems suffer from low reliability due to frequent component failures of commodity hardware. Due to the series reliability model, increasing the number of nodes or processors in an HPC 
cluster reduces the overall reliability of the whole cluster. This results in frequent failures that hinder the performance and scalability of large-scale systems.

The work in this thesis provides a small-scale fault injection system based on system logs from real HPC systems under a typical workload that are used to emulate failures on a VM-level to create a simulation of a faulty cluster for a testbed for parallel applications. This would allow parallel application developers to test their code's robustness and tolerance of node failures in a small-scale environment that replicates the failure patterns of large-scale systems. Researchers will be able to submit more fault-tolerant parallel applications to large-scale HPC systems first, instead of submitting jobs that have not been properly tested for robustness and resilience.

\subsection{Research Question}

Can a small-scale fault injection system based on real HPC system logs be used to create a suitable testbed for parallel applications?

\subsection{Assumptions}

The assumptions for this study include:

- The filtering of the system logs by RCAC did not remove any important or relevant messages.

- The system logs provided by RCAC are accurate and correct.

- MathWave's EasyFit software correctly and accurately fits datasets to statistical distributions.

- All virtual cluster nodes' system clocks were in synchrony with each other throughout the logging period. 
- The overhead of the developed system will be minimal enough to avoid significant performance penalties.

\subsection{Limitations}

The limitations for this study include:

- The developed system was tested in a virtual environment using the QEMU-KVM hypervisor only.

- The developed system was tested using the CentOS 7.2 Linux distribution only.

- The developed system was run on Intel processors.

- The developed system cannot reliably inject multiple hard disk errors when the time between errors is some time under one minute.

\subsection{Delimitations}

The delimitations for this study include:

- This study cannot examine the most critical of failure events, the machine check exception (MCE), because of an unpatched bug in the Linux kernel's handling of MCEs. This limitation applies to Linux kernel version 2.6.18.

- The studied HPC systems were manufactured by HP, use AMD processors, and run Red Hat Enterprise Linux 5.5.

- The developed system was not be tested on live HPC cluster nodes or with real HPC jobs running. 


\section{$\underline{1.7}$ Definitions}

In the broader context of thesis writing, we define the following terms:

- checkpoint-restart: a fault tolerance mechanism designed to back up the state of a system (Hacker, Romero, \& Carothers, 2009)

- component failure: any defect or error that occurs in a single computational node

- machine check architecture (MCA): the platform independent framework for detecting, reporting, and handling machine check errors ("AMD64 Architecture Programmer's Manual Volume 2: System Programming", 2011)

- machine check error: (commonly: machine check) a correctable or uncorrectable hardware error detected by the machine check architecture (MCA) ("AMD64 Architecture Programmer's Manual Volume 2: System Programming", 2011)

- machine check exception (MCE): a machine check error that cannot be corrected and frequently results in a processor's context becoming corrupted ("AMD64 Architecture Programmer's Manual Volume 2: System

Programming", 2011)

- mean time between failures (MTBF): the mean time elapsed between consecutive failure events that includes the repair/maintenance time

- mean time to failure (MTTF): the mean time elapsed from a node being added to a cluster to its first recorded failure event

- memory module: a hardware component composed of volatile random access memory (RAM) that is used as the primary cache for a node; nodes usually have many memory modules. 
- spatial locality: the apparent clustering of failure events with respect to physical node location (e.g. server rack), indicating failure event dependence on an external factor (Hacker et al., 2009)

- temporal locality: the apparent clustering of failure events with respect to time, indicating failure event interdependence (Hacker et al., 2009)

- Unix epoch timestamp: the count of the number of seconds since 1970-01-01T00:00:00 UTC

\subsection{Summary}

This chapter has provided the background information, research question, and the scope of the thesis. The next chapter provides a review of the literature related to HPC system logs and system reliability. 
CHAPTER 2. REVIEW OF RELEVANT LITERATURE

This chapter provides a review of the literature relevant to HPC system logs and HPC system reliability.

\subsection{HPC System Reliability}

Improving the reliability of high performance computing (HPC) systems is one of the leading research areas in the HPC field. Many studies have been performed that have covered various methods for understanding how, why, and when failures occur in large-scale HPC systems (Atif \& Strazdins, 2009; DeBardeleben, Blanchard, Fu, Guan, \& Zhang, 2011; Fu \& Xu, 2007; Hacker et al., 2009; Oliner \& Stearley, 2007; Pandit, Kalbarczyk, \& Iyer, 2009; Romero, 2010; Salfner \& Tschirpke, 2008; Zhang, Squillante, Sivasubramaniam, \& Sahoo, 2004; Zheng, Lan, Park, \& Geist, 2009; Zhou, Zhan, Meng, Xu, \& Zhang, 2010; Zhou, Zhan, Meng, \& Zhang, 2010).

HPC system logs are in invaluable tool for studying ways of improving the reliability of HPC systems. Unfortunately, the system logs on HPC systems are frequently of poor quality - vague, cryptic, not easily machine parsible, etc.-which necessitates significant processing to produce useful data. A notable flaw in the 2.6.18 version of the Linux kernel - the same kernel version used in Red Hat Enterprise Linux (RHEL) 5-prevents the most severe of hardware failures from being logged at all (Pandit et al., 2009). This further complicates system log analysis on clusters running RHEL 5.

Zheng et al. (2009) presented a framework for pre-processing HPC system logs to make these logs better suited for statistical analysis and failure prediction. The framework consists of three components: 
1. classifying messages by type and severity

2. removing temporal and spatial clustering of repeated messages for the same event

3. identifying causal relationships between system log messages to better identify symptomatic messages

Zheng et al. (2009) stated that their framework improved failure prediction over the incumbent mechanism by $20 \%$ to $170 \%$.

Salfner and Tschirpke (2008) proposed a set of techniques for processing the system logs of a commercial telecommunication system, and their techniques consisted of the following:

- classifying "messages based on Levenshtein's edit distance"

- clustering related error messages

- "a statistical noise filtering algorithm"

Salfner and Tschirpke (2008) stated that their techniques significantly improved upon other failure prediction methods, and the authors concluded that appropriate pre-processing of the system logs is vital for producing useful data.

Hacker et al. (2009) concluded in their analysis of system logs from two large IBM Blue Gene systems that system log messages show significant spatial and temporal clustering, that the failure events rate varies in time following the Weibull distribution, and that the health and reliability of individual computational nodes can be estimated by system log analysis.

The work in this thesis partially aims to support the live migration of virtual machines atop a cloud-like infrastructure as a service (IaaS) platform- such as OpenNebula - with which a statistical model of previous HPC system failures, to be combined in the future with the discrete-time, semi-Markov model introduced by Hacker et al. (2009), will trigger live migrations as node health decreases. An ideal 
implementation of this work, under perfect conditions, would result in improved uptime for jobs because virtual machines would be live migrated off of unhealthy hardware before failures occur.

\subsection{HPC, Cloud Computing, and Virtualization}

Cloud computing is a technology still in its adolescence, and it was only in the mid-2000s that cloud computing and virtualization exploded in popularity. Virtualization is required for almost any cost-effective cloud computing environment; thus, for cloud computing to be viable for HPC applications, the overhead introduced by virtualization must be minimal. Youseff, Wolski, Gorda, and Krintz (2006) demonstrated that the Xen virtual machine monitor (VMM) adds "no statistically significant overhead" for standard HPC benchmarks run on the small-scale HPC system tested in their study. Youseff et al. (2006) stated that their study showed that previous skepticism of virtualization for HPC applications was "unwarranted" and that the many added benefits of virtualization outweigh the negligible performance overhead.

Evangelinos and Hill (2008) stated that Amazon's Elastic Compute Cloud (EC2) is a promising platform for HPC applications because research institutions will not need to build and maintain their own HPC clusters. Evangelinos and Hill (2008) stated, however, that coupled HPC applications do suffer from a significant performance penalty incurred by the inherent design of Amazon's EC2 because Amazon does not provide clients the ability to deploy virtual machines at a granular level. Despite the performance penalty, the low cost and ability to deploy HPC applications quickly with few requirements for local IT infrastructure demonstrate the viability of cloud-based HPC systems in the future, especially if a cloud system was designed with HPC applications in mind (Evangelinos \& Hill, 2008).

The live migration of virtual machines is a process which involves copying the running virtual machine's memory pages to another physical host also running a 
VM and, in the case of Xen, iteratively copies the memory pages up to 30 times before pausing the running operation of the VM, resuming the VM on the new host, and then destroying the original VM instance (Atif \& Strazdins, 2009). Atif and Strazdins (2009) hypothesized that the overall performance of live migrations could be significantly improved by reducing the amount of CPU intensive and network intensive memory copy iterations to the bare minimum of two iterations. Atif and Strazdins (2009) demonstrated that optimizing this process resulted in a reduction of memory page transfer by over $500 \%$ for the very memory intensive HPC benchmarks. Their optimization showed improvements of almost $50 \%$ in total wall clock time spent in the live migration process and around 200\% performance improvement for memory intensive and network intensive jobs (Atif \& Strazdins, 2009). Additionally, live migration has already been shown to be a promising proactive fault tolerance technique. Romero (2010) demonstrated a reduction in the failure rate of parallel applications by live migrating multiple OpenVZ containers - basically, advanced derivatives of FreeBSD Jails - to multiple nodes that are less prone to failure.

\subsection{Error Injection}

Several error and fault injection systems have been developed previously. Giuffrida, Kuijsten, and Tanenbaum (2013) created a fault injection tool for software programs. Their system injects software faults in a controlled manner and "offers strong guarantees" that attempted software fault injections do not cause unintended side effects that could undermine the results. However, their approach requires changes be made to the tested software at compile-time or using a disassembler if source code is not available.

Guan, DeBardeleben, Blanchard, and Fu (2014) created a fault injector tool that uses the QEMU virtual machine and can precisely target programs running inside a VM. Their system is intended for introducing errors in software programs 
by modifying the program's memory by exploiting how QEMU translates virtual machine instructions between the guest and host systems. Their system exploits the Tiny Code Generation (TCG) system used by QEMU and "translates" the intended VM instructions to corrupted ones before the host executes them.

Error injection into virtual machines has been investigated previously by DeBardeleben et al. (2011). DeBardeleben et al. (2011) introduced a framework for injecting errors that evaluates the "resilience" of parallel applications to such errors. The approach DeBardeleben et al. (2011) took is targeted solely at causing faults inside the application's memory.

Levy, Dosanjh, Bridges, and Ferreira (2013) created a VM-based fault injection tool using the Palacios virtual machine monitor (Lange et al., 2011), which is a VMM developed for HPC systems. Their approach injects memory errors at specific memory locations and IDE disk errors at the VM level. Their method of injecting errors differs from our approach due to differences in the hypervisor and due to our approach being more generalized and not necessitating physical memory or disk addresses.

The work in this thesis is evaluated by testing the injection of machine checks and simulation of disk failures into a virtual cluster - in other words, causing the virtual hardware to experience similar failures and errors as those observed in real system logs under typical workloads. The work in this thesis uses libvirt (libvirt Virtualization API, 2015) - a virtualization API - and a custom QEMU to inject errors into the virtual hardware which causes the virtual machine to detect and report these errors using the inherent failure detection mechanisms within the OS.

Our approach differs from Giuffrida et al. (2013), Guan et al. (2014), and DeBardeleben et al. (2011) by simulating errors at the virtual hardware level and using the inherent failure detection mechanisms within the OS to detect the errors. Our approach improves upon the work by Levy et al. (2013) by triggering whole-disk errors which ensures that errors are always detected given some disk activity, supporting a larger array of memory and disk error types, and by using a 
mainstream virtual machine monitor in QEMU — a key ease-of-use requirement for a testbed system not intended to be deployed on real HPC systems, and therefore not bound by the same performance requirements of real HPC jobs.

\subsection{Summary}

This chapter provided an overview of the literature related to HPC system logs and HPC system reliability. The next chapter provides the research approach and methodology for the thesis. 


\section{CHAPTER 3. FRAMEWORK AND METHODOLOGY}

This chapter provides the framework and methodology that was used in this research project.

\subsection{Study Design}

This study was a quantitative research analysis of system logs from real HPC systems. This study created statistical models of component failures within the systems and used the models to simulate failure events on virtual machines. The virtual machines experienced failures similar to those found in the logs from real HPC systems.

\subsubsection{Data Sets}

Memory error log data was taken from system logs from the 993-node Coates system (Rosen Center for Advanced Computing, n.d.-b) operated by the Rosen Center for Advanced Computing (RCAC) at Purdue University. The logs analyzed ranged from 2009-09-01 to 2011-02-20.

Hard disk error log data was taken from system logs from the 660-node Carter system (Rosen Center for Advanced Computing, n.d.-a) operated by RCAC at Purdue University. The logs analyzed ranged from 2012-11-25 to 2013-08-31.

The Coates and Carter systems were operating under typical HPC workloads throughout the log collection period. This makes the analyzed log data generalizable to other large-scale systems operating under workload. 


\subsubsection{Overview of Statistical Model Creation}

This section provides an overview of the steps taken to create the statistical models. This is provided to assist other researchers that may be interested in creating statistical models for errors on other systems.

The system logs for each system were collected in a central location and were stripped of personally-identifiable information prior to the beginning of this study.

The following list provides a high-level overview of the procedure.

\section{Error discovery}

2. Error filtering

3. Error sorting

4. Log message timestamp sanitization

5. Scatter plot creation to identify clustering of errors

6. Declustering data using k-means

7. Fitting distributions to the data

8. Selecting a model from the fitted distributions

Manual searching of the system logs was performed using grep with regular expressions to look for anomalous log events. Error messages of interest were then filtered out from the rest of the logs and sorted based on the type of error. Due to poor quality timestamps of the Coates system logs, these timestamps had to be corrected prior to further processing.

Scatter plots were made of the error event data to identify the severity of clustering. We then declustered the data using k-means cluster analysis. The declustered data was loaded into distribution fitting software. Finally, the continuous statistical distributions with the best fit were selected as our models. 


\subsubsection{System Log Analysis}

The system logs were manually examined to determine the key phrases within the logs related to failure events of memory and hard disks. The logs were searched using a grep regular expression like the one below:

grep -Ei error messages*

The system logs from Coates needed significant processing to improve the quality of message timestamps. The system logs from Carter did not need any notable processing.

\subsubsection{Coates: Log Processing and Sorting}

The logs were filtered so that only failure-event-related messages were kept. The logs were further processed to identify specific failure messages and were sorted by those messages.

We investigated general errors detected by the integrated memory controller - an external memory controller is called a northbridge, but this distinction is not made in the system logs. The "BIOS and Kernel Developer's Guide for AMD Athlon 64 and AMD Opteron Processors" (2006) states that these errors include errors in the GART TLB cache, errors in the HyperTransport link, or errors in DRAM. It should be noted that the RHEL kernel version 2.6.18-194.17.1.el5 disables GART TLB error reporting by default. The "node" and "core" values mentioned below refer to the physical CPU package (i.e. socket) and the CPU core number within the physical CPU package, respectively. Because the Coates cluster is built entirely of dual-socket, quad-core AMD Opteron processors, the only valid values for "node" are 0 and 1 , and the only valid values for "core" are $0,1,2$, and 3 .

The regular expression used to match these errors is

kernel:. ${ }^{*}$ Northbridge Error

and an example log message is 
Feb 16 14:45:50 172.18.42.117 kernel: Northbridge Error, node 1, core: 2

\subsubsection{Coates: Timestamp Standardization}

The timestamps in the Coates logs were changed from the default BSD-style syslog format, specified in RFC 3164, to the Unix epoch format for easier machine processing. Table 3.1 compares the differences between the easily human-readable ISO 8601 and BSD-style syslog formats and the easily machine-readable Unix epoch format for a sample from the Coates system logs. For the BSD-style syslog format, the year was determined based on log file metadata because the year is not included in these timestamps.

Table 3.1

Comparison of various timestamp formats

\begin{tabular}{cc}
\hline Format & Example timestamp \\
\hline ISO 8601 & 2011-02-13T09:02:39Z \\
BSD-style syslog & Feb 13 04:02:39 \\
Unix epoch & 1297587759 \\
\hline
\end{tabular}

\subsubsection{Coates: Node ID Standardization}

To look for temporal and spatial clustering of events, we created a scatter plot to investigate the degree of clustering. To plot the nodes on the horizontal axis, the nodes were assigned unique IDs based on their IP addresses.

The unique node IDs were generated based on the compute node's IP address. The IP address was converted from dotted decimal notation, e.g. 192.168.15.76, into its packed, 32-bit, binary format represented as a decimal, e.g. 3232239436. Sequential node IDs were then generated by sorted the decimal-based node IDs. The data set, composed of unique node IDs and the message timestamps 
of failure events, was plotted as a scatter plot with the node IDs sorted from smallest to largest along the horizontal axis and the event timestamps plotted along the vertical axis.

\subsubsection{Coates: Scatter Plots of Memory Failure Events}

Figure 3.1 shows a scatter plot of memory failure event times plotted against node IDs.

\subsubsection{Coates: Declustering of Failure Events}

Notable temporal and spatial clustering of events was found in the data set, as shown in Figure 3.1. The temporal and spatial clustering of events shows that these events are not randomly distributed. This can be for a number of various reasons including faulty hardware, physical location in the data center, etc. The events for memory errors were declustered by Rui Máximo Esteves from the University of Stavanger in Norway using the R statistical software (Esteves, Hacker, \& Rong, 2012).

\subsubsection{Carter: Scatter Plots of Hard Disk Failure Events}

Figure 3.2 shows a scatter plot of hard disk failure event times plotted against node IDs.

\subsubsection{Carter: Declustering of Failure Events}

Notable temporal and spatial clustering of events was found in the data set, as shown in Figure 3.2. The events for hard disk errors were declustered using the R statistical software (The $R$ Project for Statistical Computing, 2016) by the author. The "neural gas" k-means algorithm was used from the "cclust" package in R. The 


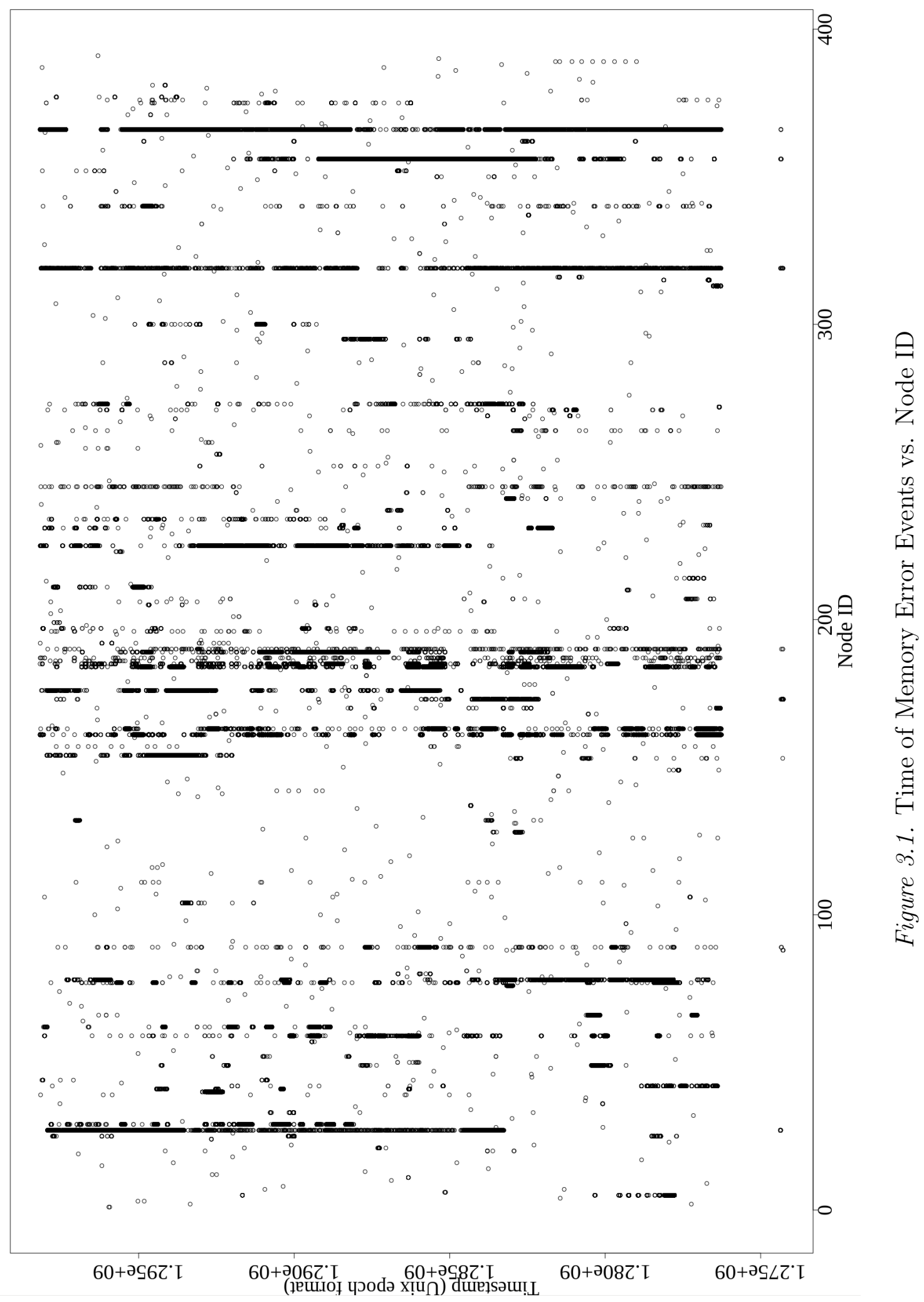




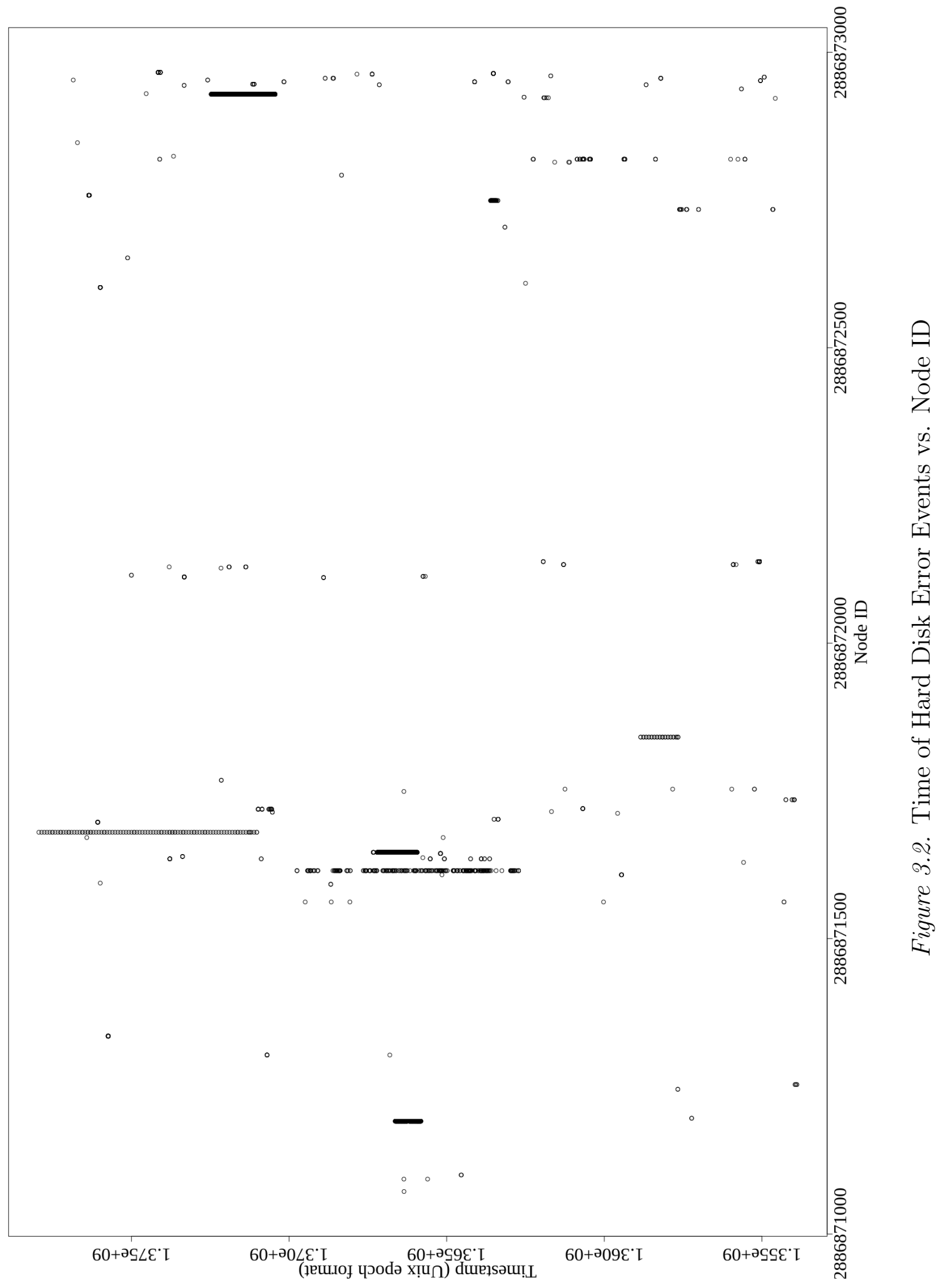


number of centers was chosen by locating the "elbow" of the plot of within groups sum of squared error (WSSE) versus the number of centers. WSSE is a measure of the accuracy of the k-means fit. WSSE is the sum of the distance squared between data points and their assigned cluster. When the slope of a polynomial fitted to a plot of the WSSE vs. the number of centers approaches 0 , then the optimum number of centers has been found because increasing the number of centers does not lower WSSE any further.

The elbow was located by fitting a fifth-degree polynomial to the WSSE plot using MatLab and finding the real roots of the polynomial. The polynomial, in Equation 3.1, has the coefficients shown in Table 3.2:

$$
p 1 * x^{5}+p 2 * x^{4}+p 3 * x^{3}+p 4 * x^{2}+p 5 * x+p 6
$$

Table 3.2

Polynomial coefficients

\begin{tabular}{cr}
\hline Coefficient & Value \\
\hline $\mathrm{p} 1$ & $-3.204 \mathrm{e}-07$ \\
$\mathrm{p} 2$ & 0.005271 \\
$\mathrm{p} 3$ & -34.83 \\
$\mathrm{p} 4$ & $1.157 \mathrm{e}+05$ \\
$\mathrm{p} 5$ & $-1.934 \mathrm{e}+08$ \\
$\mathrm{p} 6$ & $1.31 \mathrm{e}+11$ \\
\hline
\end{tabular}

Differentiating the polynomial and solving for its roots in MatLab produced the solutions shown in Table 3.3. The first real root was selected and rounded down to 3197 . K-means was run using 3197 centers to get the final declustered data set for Carter. Figure 3.3 shows a plot of WSSE vs. the number of clusters with a clear "elbow".

Table 3.4 lists the number of centers vs. WSSE. 
Table 3.3

Polynomial roots

$\begin{array}{r}\text { Roots } \\ \hline 3197.3+0.0000 \mathrm{i} \\ 3762.3+0.0000 \mathrm{i} \\ 3100.7-0.6492 \mathrm{i} \\ 3100.7+0.6492 \mathrm{i} \\ \hline\end{array}$

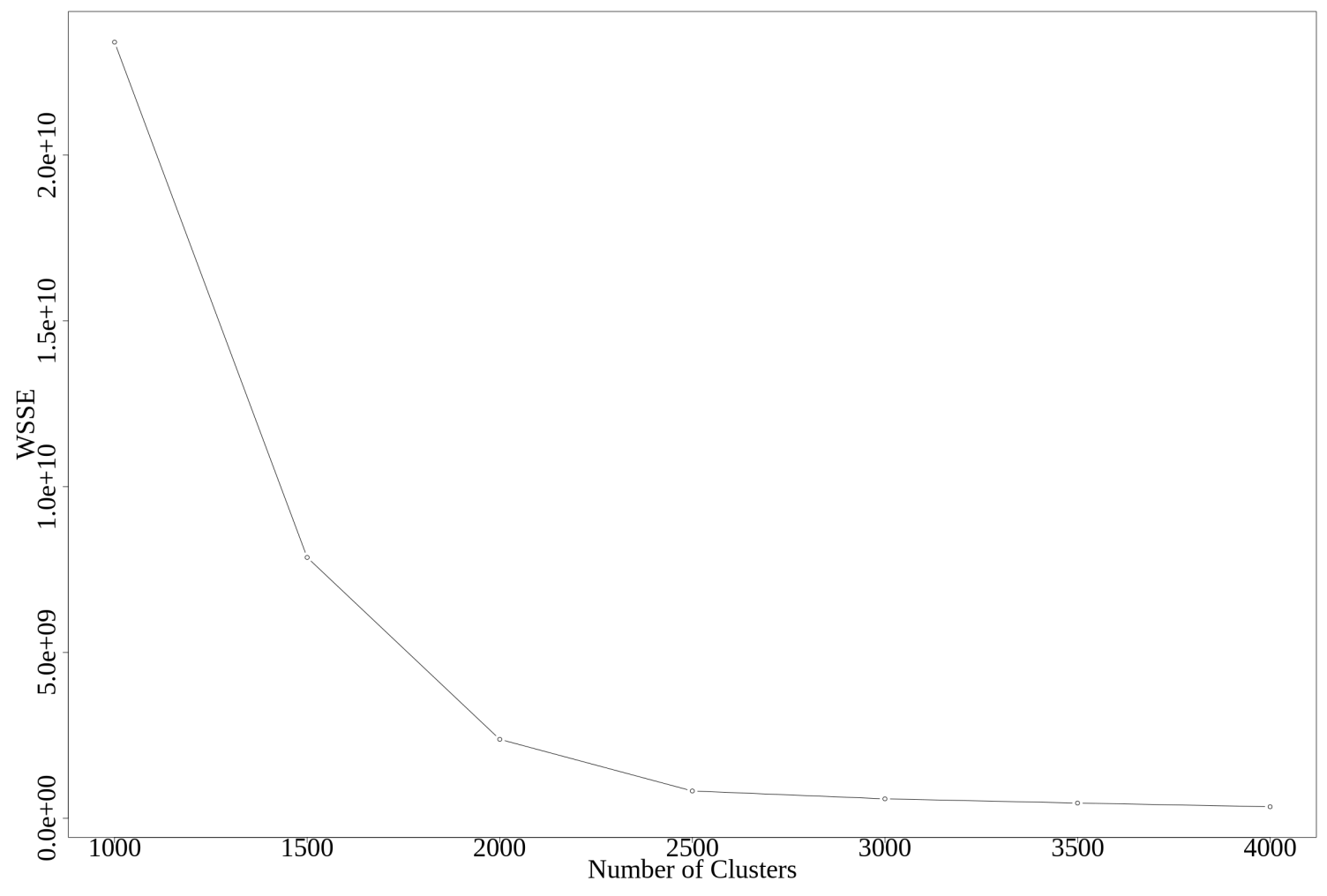

Figure 3.3. WSSE vs. Number of Clusters 
Table 3.4

Number of centers vs. WSSE

\begin{tabular}{rr}
\hline Number of Centers & WSSE \\
\hline 1000 & 23408200075.0000 \\
1500 & 7866952504.0000 \\
2000 & 2380480559.0000 \\
2500 & 823117829.2000 \\
3000 & 585667433.3000 \\
3500 & 458904640.7000 \\
4000 & 347255632.8000 \\
\hline
\end{tabular}

\subsubsection{Distribution Fitting}

The declustered data sets for Coates and Carter were then input into MathWave's EasyFit software for distribution fitting (EasyFit, 2010). The fit process was run using a continuous data domain of the time between events data. The distributions with the best goodness of fit results were chosen.

\subsubsection{Fault Injection}

We created a Python 3 script that can inject arbitrary machine checks into VMs and simulate hard disk failures for virtual disk drives. Machine checks were injected using QEMU's built-in Human Monitor Protocol (QEMU Emulator User Documentation: QEMU Monitor, n.d.). Hard disk errors were simulated using modified VirtIO SCSI drivers for QEMU (Bellard, 2014).

The script has a multi-threaded design that allows for an arbitrary number of VMs to be supported. When an error is about to be injected, the script randomly selects the VM in which to inject the error from a preconfigured list of VMs. Appendix A contains the source code for our fault injection script. 


\subsubsection{Memory Error Injection Procedure}

Arbitrary memory errors are injected using QEMU's Human Monitor Protocol (HMP). The HMP has various commands that can be executed via libvirt's "virsh" tool. One of these commands is the "mce" command that sets the values of the virtual CPU's $M C i_{-} S T A T U S$ and related registers. These registers control the Machine Check Architecture (MCA) subsystem of the processor, which is used for memory and processor error detection and reporting.

For example, running the following command will inject an ECC error overflow with a valid address into CPU 0 bank 4 of domain 12:

virsh -c qemu:///system qemu-monitor-command 12 -hmp-cmd 'mce 04 0xd426c0010b000813 0x0 0x0 0x0'

\subsubsection{Machine Check Registers}

The memory error messages analyzed were composed of errors detected in the on-die northbridge of the systems' AMD processors. These memory errors are handled by the processor's Machine Check Architecture, which contains several registers indicating the type of error detected by the processor. The $M C i_{-} S T A T U S$ register is the register that reports the error detected. The underlying bit patterns of the $M C i_{-} S T A T U S$ register were analyzed based on AMD's system programming manuals ("AMD64 Architecture Programmer's Manual Volume 2: System Programming", 2011; "BIOS and Kernel Developer's Guide for AMD Athlon 64 and AMD Opteron Processors", 2006), the Linux kernel documentation (Thompson, Jiang, Peterson, Harbaugh, \& Chebab, 2011), and the RHEL kernel's source code (Torvalds, Red Hat, Inc., et al., 2010). The bit patterns required to inject the observed failure events were recorded and added to the fault injection system. 


\subsubsection{Memory Error Injection Strings}

The fault injection system supports injecting ten types of memory errors. Table 3.5 documents the supported memory errors. Uncorrectable errors are indicated by "(UE)". Overflow errors occur when more than one machine check occurs within the machine check polling interval.

Table 3.5

Supported memory errors and $M C i_{-} S T A T U S$ register values

\begin{tabular}{ll}
\hline Error Type & MCi_STATUS Register \\
\hline ECC error (ADDR valid) & 0x9426c0010b000813 \\
ECC error overflow (ADDR valid) & 0xd426c0010b000813 \\
ECC error (ADDR invalid) & 0x9026c0010b000813 \\
ECC error overflow (ADDR invalid) & 0xd026c0010b000813 \\
L1 Cache Data Store error (UE) & 0xb600200000000145 \\
L1 Instruction Cache (Instruction Fetch) & \\
$\quad$ error (ADDR valid) & 0x9400000000000151 \\
L1 Instruction Cache (Instruction Fetch) & 0xd400000000000151 \\
$\quad$ error overflow (ADDR valid) & 0xb600000000020136 \\
Bus Unit (L2 Cache) error (UE) & 0x9400400000000136 \\
L2 Data Cache (Line Fill) error (ADDR valid) & 0xd400400000000136 \\
L2 Data Cache (Line Fill) error overflow (ADDR valid)
\end{tabular}

\subsubsection{QEMU Modifications and Hard Disk Error Simulation Procedure}

QEMU does not support disk error injection by default. We modified the source file $\mathrm{hw} / \mathrm{scsi} / \mathrm{scsi}$-disk.c

from QEMU version 2.0.0 to support disk error injection via the VirtIO SCSI subsystem. When a hard disk error is triggered and detected, the VM's SCSI driver 
skips to its built-in error handling code and propagates the error to the rest of the system using the OS's inherent failure detection mechanisms. Appendix B contains the source code changes we made.

Hard disk errors are injected by the existence of a file under a directory tree containing MAC addresses that map the errors to specific VMs. For example, the existence of the following file will simulate the error ENOMEDIUM for the VM with MAC address 52:54:00:39:ca:8b:

/tmp/qemu-disk-inject/52:54:00:39:ca:8b/ENOMEDIUM

The Flexible I/O Tester (fio) (Axboe, 2015) was used to simulate a light disk I/O workload on the VMs. This ensures that the injected errors are recognized by QEMU and the guest OS.

\subsubsection{Hard Disk Errors}

The fault injection system supports injecting four types of hard disk errors; however, only the errors ENOMEM, ENOMEDIUM, and EINVAL were used for the simulations. Injecting the error ENOSPC immediately suspends the VM, making this error not suitable for simulations. These four errors are all of the errors supported by QEMU. Table 3.6 documents the supported errors and their descriptions. The descriptions are from

$$
\text { /usr/include/asm-generic/errno\{,-base }\} . h
$$

Table 3.6

Supported hard disk errors and their descriptions

\begin{tabular}{ll}
\hline Errno & Description \\
\hline ENOMEM & out of memory \\
ENOSPC & no space left on device \\
ENOMEDIUM & no medium found \\
EINVAL & invalid argument \\
\hline
\end{tabular}




\subsubsection{Tunable Fault Injection Frequency}

The script supports tunable fault injection frequency. The statistical distributions from EasyFit were mathematically analyzed to allow for arbitrary changes to the expected values of the distributions. The scale parameters to the distributions were made variable, via an inverse multiplier term, to provide a desired increase or decrease in the frequency of failure events, similar to a tuning knob.

\subsubsection{Tuning the Three-Parameter Weibull Distribution}

Integrated memory controller errors on the Coates system followed a three-parameter Weibull distribution with the parameters found in Table 4.1. The expected value of the three-parameter Weibull distribution is shown in Equation 3.2, where $\Gamma$ is the Gamma function, $\gamma$ is the shape parameter, $\alpha$ is the scale parameter, and $\mu$ is the location parameter (NIST, 2003).

$$
\mathbf{E}[X]=\Gamma\left(1+\frac{1}{\gamma}\right) * \alpha+\mu
$$

Rearranging the terms of Equation 3.2 gives a formula for an alternate scale parameter with an inverse multiplier term, as shown in Equation 3.3, where $\mathbf{E}[X]$ is the expected value and $\beta$ is the multiplier.

$$
\alpha=\frac{\left((\mathbf{E}[X]-\mu) *\left(\frac{1}{\beta}\right)\right)}{\Gamma\left(1+\frac{1}{\gamma}\right)}
$$

\subsubsection{Tuning the Lomax Distribution}

Hard disk errors on the Carter system followed a Lomax distribution-Pareto Type II distribution that starts at 0 - with the parameters found in Table 4.2. The expected value of the Lomax distribution is shown in Equation 3.4, where $\lambda$ is the scale parameter, and $\alpha$ is the shape parameter.

$$
\mathbf{E}[X]=\frac{\lambda}{\alpha-1}
$$


Rearranging the terms of Equation 3.4 gives a formula for an alternate scale parameter with an inverse multiplier term, as shown in Equation 3.5, where $\mathbf{E}[X]$ is the expected value and $\beta$ is the multiplier.

$$
\lambda=\mathbf{E}[X] * \frac{1}{\beta} *(\alpha-1)
$$

\subsubsection{Experimental Setup}

The fitted distributions were then used as input for the fault injection script to inject errors with the same statistical distributions observed in the Coates and Carter logs.

The testbed server is a custom-built, dual-socket Intel Xeon X5650 system with 24 cores and 36 GiB of DDR3 memory. The stock QEMU package was installed, and our custom QEMU was compiled and installed manually into /usr/local/bin

To replicate this, future users can apply the "diff" from Appendix B to the QEMU 2.0.0 source tarball using "git apply", install the needed dependencies from the "configure" script, and then compile and install the software:

./configure make make install

The stock QEMU executable was backed up, and a symlink was created to our custom QEMU executable from

/usr/libexec/qemu-kvm

We added the SELinux tag "qemu_exec_t" to the custom QEMU executable for SELinux compatibility. Eight QEMU-KVM virtual machines were created on the testbed server. Using virt-manager, we changed their SCSI controller type from "hypervisor default" to "VirtIO SCSI", and we changed the disk bus to "SCSI" with a "raw" storage format. The server and its eight VMs run CentOS 7.2 without a GUI.

mcelog (Kleen, 2015) was installed on the guest VMs to decode the injected machine checks. The Flexible I/O Tester (fio) (Axboe, 2015) was configured as a 
workload generator on all eight VMs. fio has been configured in time-based mode scheduled for a two month duration using the Intel IOMeter File Server Access Pattern job configured with a "linear" I/O depth, as shown in Figure 3.4. A systemd service file, shown in Figure 3.5, was created to start the fio service at boot and to automatically restart on service failure. The eight VMs were configured to forward their system logs to a centralized server for easy log collection and monitoring.

A systemd service file, shown in Figure 3.6, was created to set the Linux kernel's machine check "check_interval" from 300 seconds to 1 second. Figure 3.7 shows the Bash script that is called from the ExecStart directive from Figure 3.6. This systemd service file and Bash script were installed on the VMs.

\section{$\underline{3.2 \text { Unit \& Sampling }}$}

The following sections will discuss the hypotheses, population, samples, variables, and the measure for success.

\subsubsection{Hypothesis}

The hypotheses for this study are the following:

$\mathrm{H}_{0}$ : It is not possible to develop a small-scale fault injection testbed that can emulate the types of faults on large-scale HPC systems.

$\mathrm{H}_{\alpha}$ : It is possible to develop a small-scale fault injection testbed that can emulate the types of faults on large-scale HPC systems.

\subsubsection{Population}

The population of the memory error system log data is a set of logs from the "Coates" compute cluster operated by the Rosen Center for Advanced Computing (RCAC) at Purdue University. The population of the hard disk system log data is a 


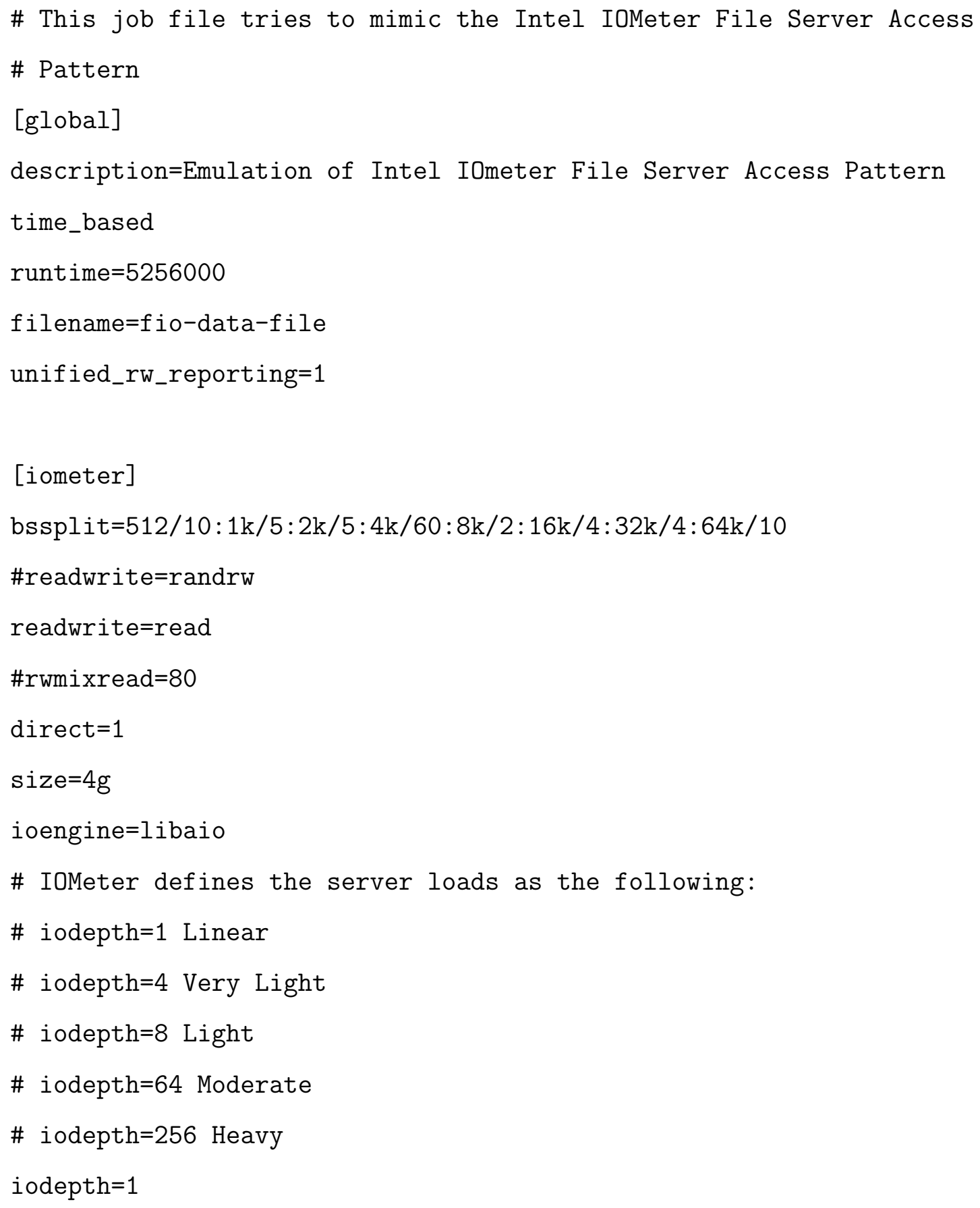

Figure 3.4. Configuration file for fio 


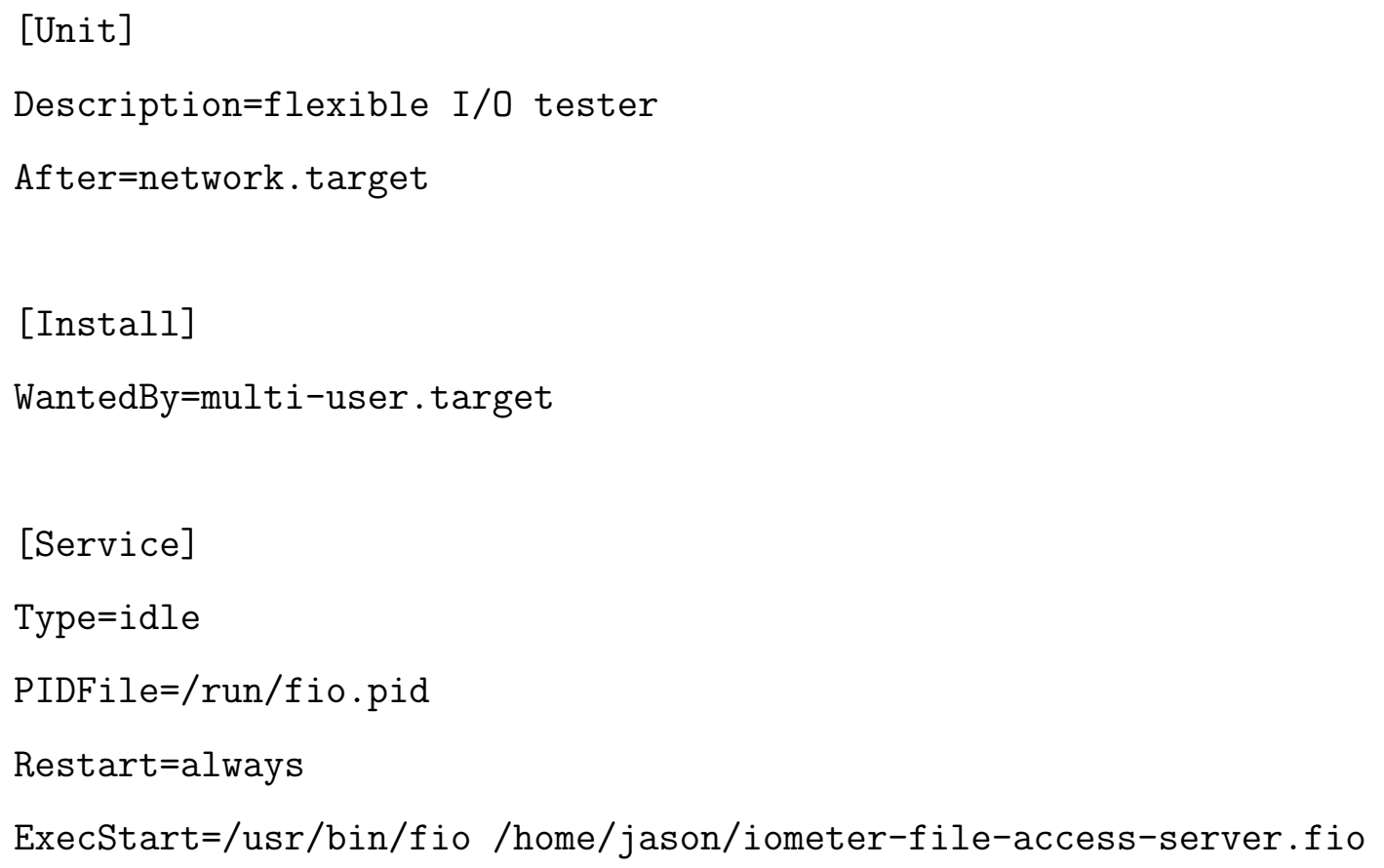

Figure 3.5. systemd service file for fio, installed on the VMs

\section{[Unit]}

Description=Change the default polling frequency for machine checks After=network . target

[Install]

WantedBy=multi-user . target

\section{[Service]}

Type=oneshot

ExecStart=/usr/bin/bash /home/jason/set-mce-polling-frequency.sh

Figure 3.6. systemd service file for changing the default polling interval for machine checks 


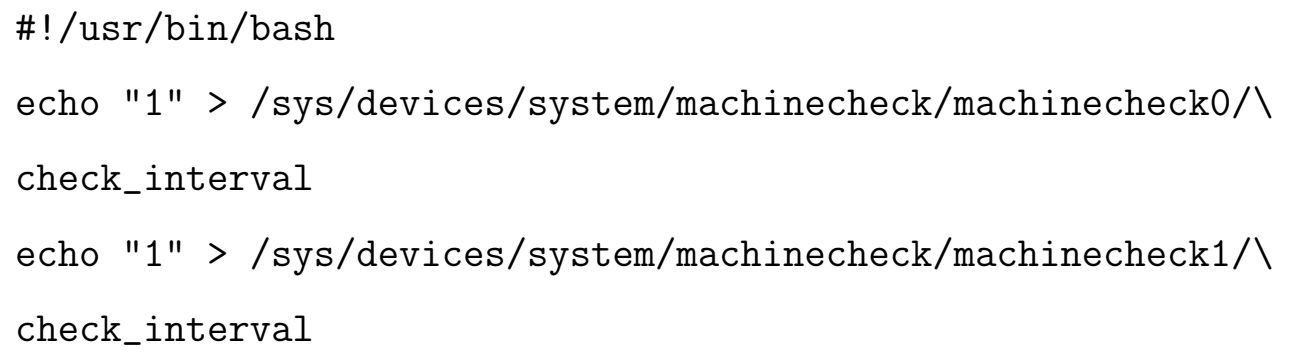

Figure 3.7. Bash script that changes the default polling frequency for machine checks 
set of logs from the "Carter" compute cluster operated by RCAC at Purdue University.

The population of the experimental units is a set of virtual machines deployed on a small set of servers.

\subsubsection{Sample}

The fault injection system uses the Python 3 Standard Library and SciPy (SciPy-Developers, 2013) to sample the statistical distributions with the specified parameters.

\subsubsection{Variables}

The independent variable was the frequency of fault injections for each failure event type. The dependent variables were the systems' perceived "healthiness" and accuracy of triggered migrations with respect to "actual" major failures.

\subsubsection{Measure for Success}

The study examined the data and tested the null hypothesis with a $95 \%$ successful injection rate.

\section{$\underline{3.3 \text { Summary }}$}

This chapter provided the research approach and methodology that was used in the execution of this research project. 


\section{CHAPTER 4. PRESENTATION OF DATA}

This chapter provides the results from this research project.

\subsection{Statistical Models}

This section describes the statistical models for failure events observed in the Coates and Carter data sets.

\subsubsection{Coates: Memory Errors}

Integrated memory controller errors on the Coates system followed a three-parameter Weibull distribution with the parameters found in Table 4.1. This correlates with the results from Hacker et al. (2009) that also showed a Weibull distribution fits well with failure events.

Table 4.1

Parameters of the three-parameter Weibull distribution for integrated memory controller errors on the Coates system

\begin{tabular}{cc}
\hline Parameter & Value \\
\hline Shape & 0.49575 \\
Scale & 5293.0 \\
Location & 9.1171 \\
\hline
\end{tabular}




\subsubsection{Carter: Hard Disk Errors}

Hard disk errors on the Carter system followed a Lomax distribution (Pareto Type II that starts at 0) with the parameters found in Table 4.2. This correlates with the results from Schroeder, Damouras, and Gill (2010) that also showed a Pareto distribution is the best fit for disk errors.

Table 4.2

Parameters of the Lomax distribution for hard disk errors on the Carter system

\begin{tabular}{cc}
\hline Parameter & Value \\
\hline Shape & 0.38278 \\
Scale & 450.36 \\
\hline
\end{tabular}

\section{$\underline{4.2 \text { Experiments }}$}

This section contains the results of the experimental setup.

\subsubsection{System Log Samples}

This section contains system log samples from the VMs running under the fault injection script.

\subsubsection{Quantitative Results}

This section contains quantitative results from the experiments comparing the number of attempted fault injections versus the number of faults the guest OS of the VMs reported.

Over a 7 day time period, 15,644 total faults were randomly injected across the eight VMs. Table 4.3 shows the number of attempted injections, the number of successful injections, and an adjusted number of injections that accounts for 


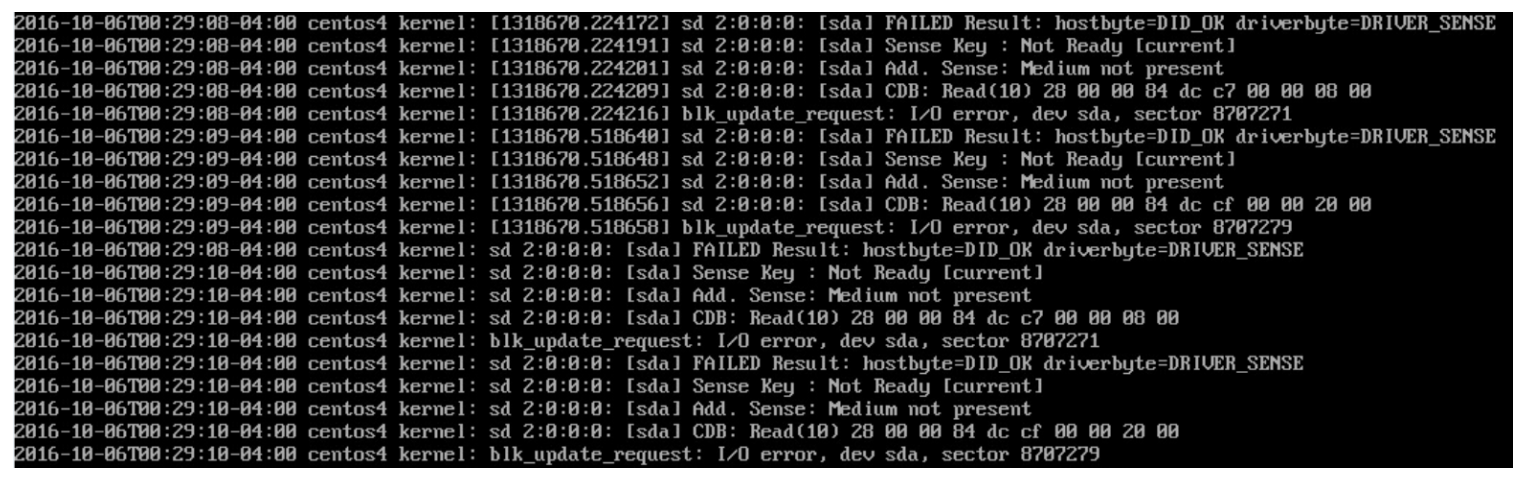

Figure 4.1. System log screenshot of VM "centos4" experiencing an ENOMEDIUM error injection 
duplicates outside the scope of this thesis due to the limitations of the hard disk injection mechanism. The adjusted value was tabulated by examining the logs on a per-VM basis and using a sliding window of one minute. When multiple disk errors occurred within one minute of each other, the first error time was noted and one minute was added to this time; any errors that occurred within this window were marked as successful and tabulated under the adjusted column. The values for ENOMEM and EINVAL have higher adjusted values than errors attempted due to uncertainty in how big this sliding window must be. From this data, $99.7 \%$ of disk errors were successfully injected; $100 \%$ of memory errors were successfully injected; and $99.99 \%$ of all total errors were successfully injected.

Table 4.3

Number of attempted and successful fault injections

\begin{tabular}{lrrr}
\hline Error & Attempted & Successful & Adjusted \\
\hline ENOMEDIUM & 300 & 182 & 298 \\
ENOMEM & 205 & 122 & 216 \\
EINVAL & 230 & 150 & 301 \\
ECC error (ADDR valid) & 143 & 143 & $\mathrm{~N} / \mathrm{A}$ \\
L1 Instruction Cache (Instruction Fetch) & 143 & 143 & $\mathrm{~N} / \mathrm{A}$ \\
$\quad$ error (ADDR valid) & & & \\
L2 Data Cache (Line Fill) error & 14623 & 14623 & $\mathrm{~N} / \mathrm{A}$ \\
$\quad$ (ADDR valid) & & & \\
\hline
\end{tabular}




\section{CHAPTER 5. CONCLUSIONS, DISCUSSION, AND RECOMMENDATIONS}

\subsection{Conclusions and Discussion}

We have processed and analyzed system log data from two high performance computing clusters running typical HPC workloads operated by the Rosen Center for Advanced Computing at Purdue University. We have produced statistical models of hardware errors in these systems. We have developed a fault injection testbed that, in virtual machines, injects/simulates real hardware errors using the same statistical models we produced from these computing clusters.

As seen in the raw injection data, a number of attempted disk error injections did not succeed. We found that hard disk errors cannot be reliably injected with a time between errors of some time under one minute. After examining the data, most of these failed error injections can be accounted for with multiple injections in short succession before the virtual machines were able to recover from previous failures. We postulate this is because the workload generator crashes at every hard disk injection, and the generator needs time to restart itself fully, along with the normal hard disk recovery procedures.

The statistical models we created are broadly applicable due to the high quality of the source data - large-scale HPC systems running typical workloads for long durations. HPC system logs of production systems under workload are rarely made available to researchers so we believe these statistical models will be useful for other researchers and system administrators that do not have access to such data.

The fault injection system we created is useful for parallel application developers and system administrators that want to test their application's robustness to hardware errors. Parallel application developers can test how their 
programs respond to errors in main memory and CPU caches, which is especially useful for highly optimized programs designed to stay in the CPU caches.

Additionally, system administrators can test before deploying to production how arbitrary web applications, customer-facing web portals like Internet-based stores, high availability software, etc. respond to failing hardware that could interrupt business functions or result in lost revenue.

\section{$\underline{5.2 \text { Recommendations }}$}

For future work, we recommend further analysis of the Rosen Center's cluster data sets for other hardware error types and generate more statistical models for these errors. We also recommend working with the QEMU project maintainers to create a built-in mechanism for hard disk error injection. Finally, we recommend extending this work by investigating error prediction based on prior hardware errors. 
APPENDICES 


\section{APPENDIX A. FAULT INJECTION SCRIPT}

\#! /usr/bin/env python3

"" "Docstring: Use QEMU and libvirt to inject arbitrary errors into VMs.

Author: Jason St. John

License: Apache 2.0

Copyright 2016 Jason St. John

Licensed under the Apache License, Version 2.0 (the "License");

you may not use this file except in compliance with the License.

You may obtain a copy of the License at

http://www . apache.org/licenses/LICENSE-2.0

Unless required by applicable law or agreed to in writing, software distributed under the License is distributed on an "AS IS" BASIS, WITHOUT WARRANTIES OR CONDITIONS OF ANY KIND, either express or implied. See the License for the specific language governing permissions and limitations under the License.

Python version required: $>=3.2$

This script generates lists of sojourn times between machine check and 
disk fault injections based on various statistical distributions and injects the given error using libvirt and a custom QEMU.

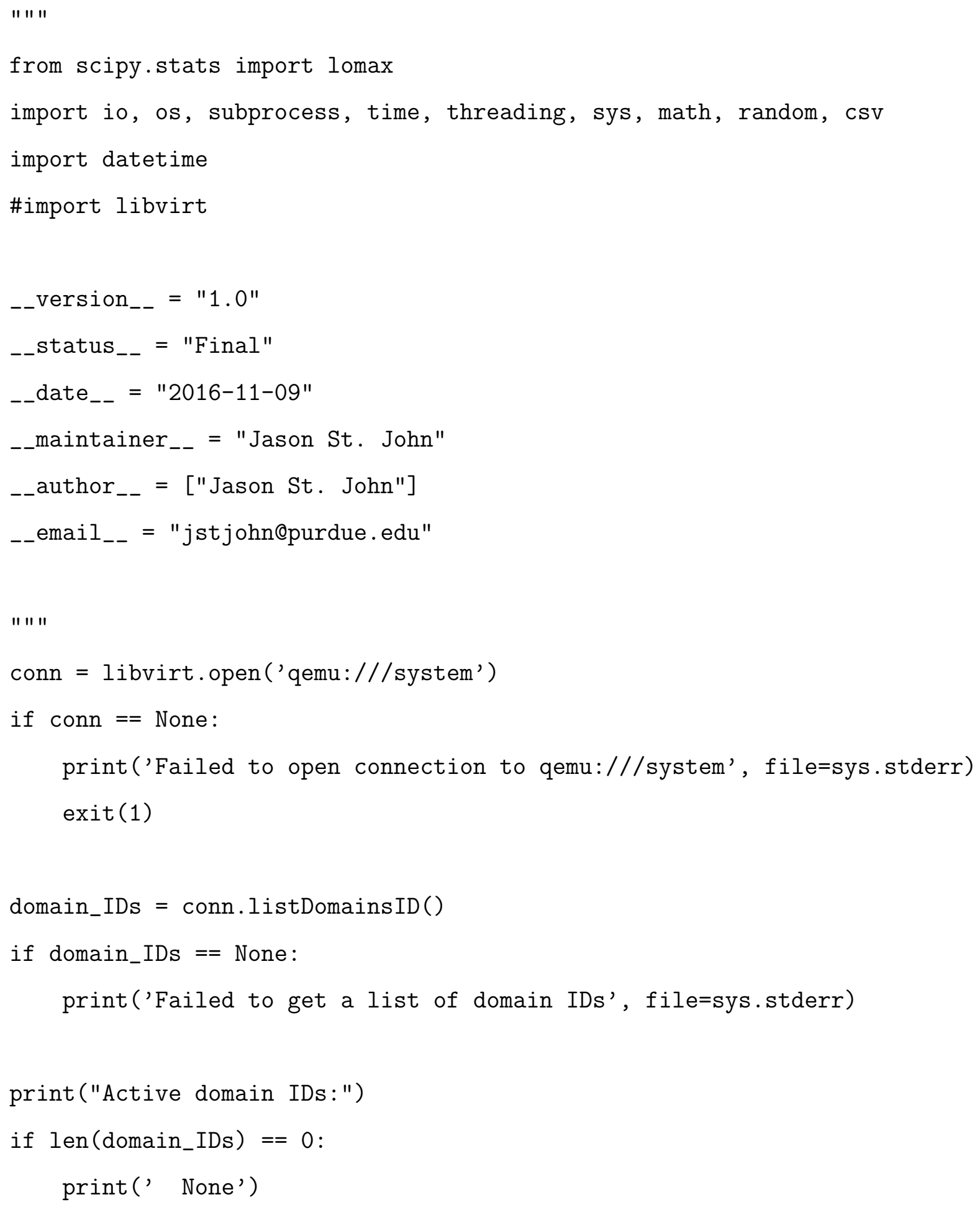


else:

for domain_ID in domain_IDs:

$\operatorname{print}\left({ }^{\prime},+\operatorname{str}(\right.$ domain_ID))

" " " "

\# These are dicts of domain (read: VM) IDs \& MAC addresses for use \# with libvirt. The "high", "normal", and "low" denote fault frequency. \# Run 'virsh -c qemu:///system list' to find the domain IDs you want \# to use. MAC addresses can be found via virt-manager or virsh. domain_list_high $=\{$

'9': '52:54:00:52:66:c1',\#centos1

'4': '52:54:00:42:6f:d4',\#centos4

'6': '52:54:00:26:57:2d',\#centos6

'7': '52:54:00:ae:f6:2a'\#centos7

\}

domain_list_normal $=\{$

'2': '52:54:00:1e:cc:65',\#centos2

'5': '52:54:00:e0:0a:6d',\#centos5

'8' : '52:54:00:82:53:05'\#centos8

\}

domain_list_low $=\{$

'3': '52:54:00:cf:de:c7'\#centos3

\}

" " " "

\# Check whether the targeted VMs are running domain_list_all = list (domain_list_high.keys ()$)$

domain_list_all += list(domain_list_normal.keys () )

domain_list_all += list(domain_list_low.keys())

for domain in domain_list_all: 
if domain not in domain_IDs:

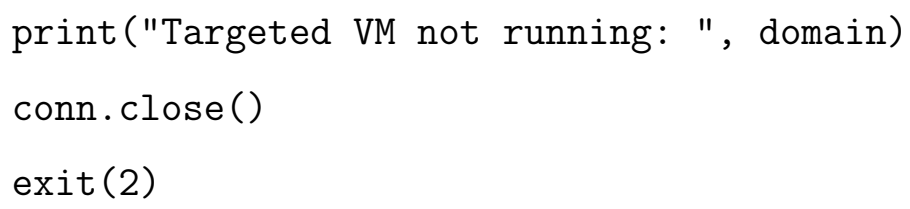


def lomax_alt_scale(mean, multiplier, shape):

"" "Return an alternate scale parameter used to tweak the intensity."" alt_scale $=$ mean $*(1 /$ multiplier $) *($ shape -1$)$

return alt_scale

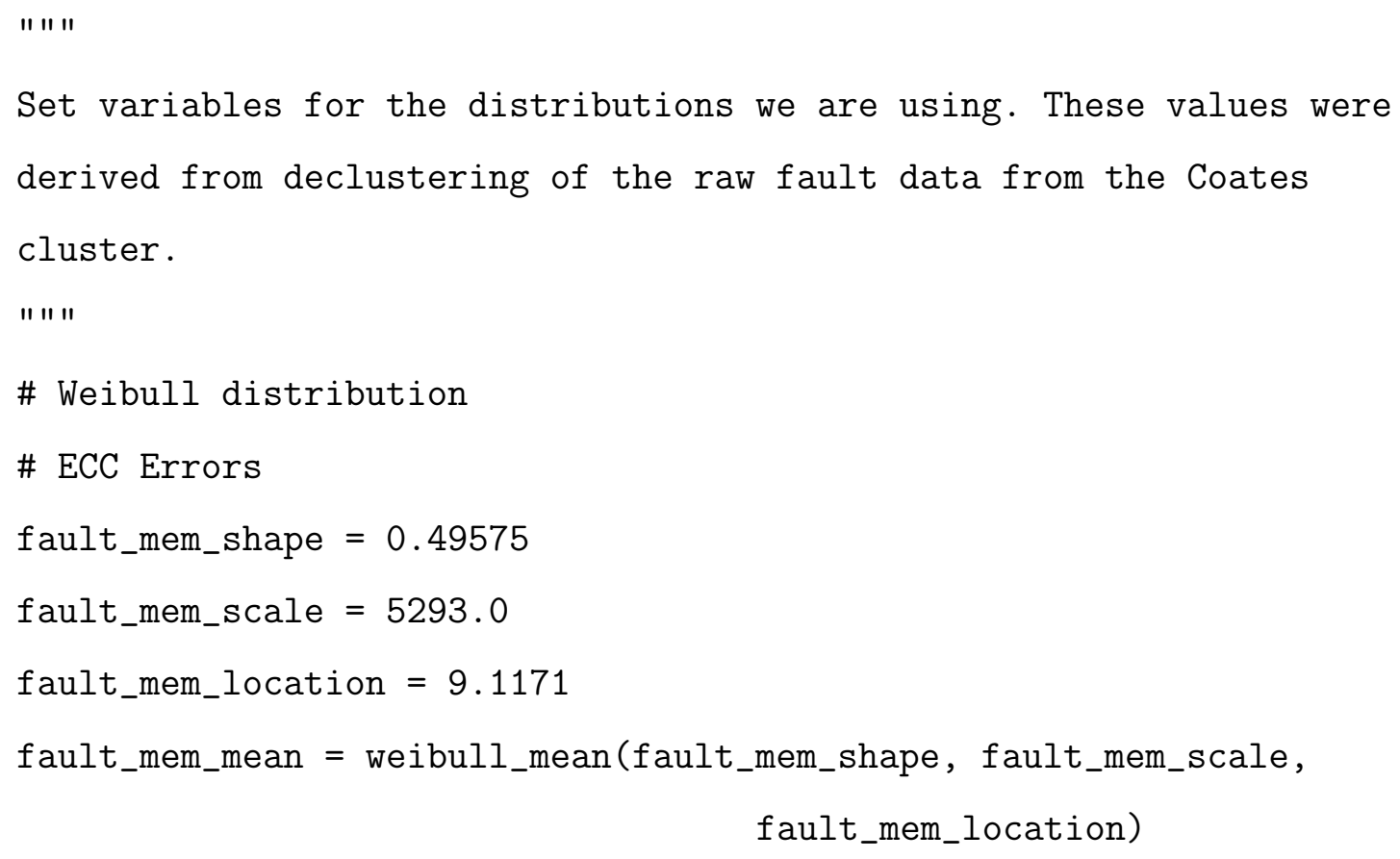




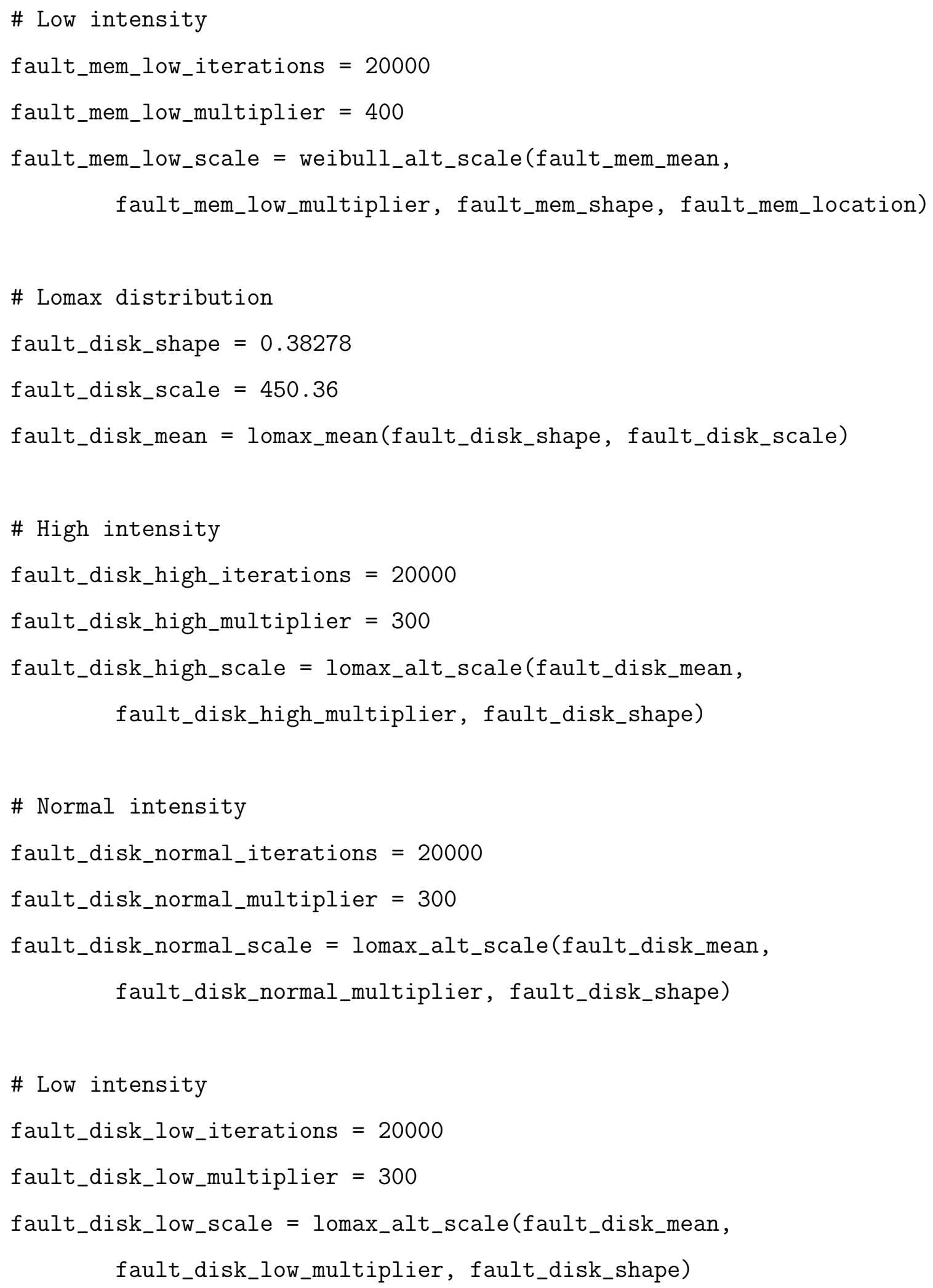


" " "

These strings contain commands for the QEMU monitor to inject arbitrary machine checks (MCs) and machine check exceptions (MCEs).

Note: '_of' designates an overflow machine check.

Format: "mce [cpu] [bank] [status] [mcgstatus] [addr] [misc]"

Reference AMD publications \#24593 and \#26094 for documentation on the format of the [status] section.

" " "

\# L2 Data Cache (Line Fill) error (ADDR valid)

mc0_inject = "mce $000 \times 94004000000001360 \times 0$ 0x0 0x0"

mc0_inject_of $\quad=$ "mce $000 x d 4004000000001360 \times 0 \quad 0 \times 0 \quad 0 \times 0 "$

\# L1 Instruction Cache (Instruction Fetch) Error (ADDR valid)

mc1_inject = "mce $010 \times 94000000000001510 \times 0$ 0x0 0x0"

mc1_inject_of $=$ "mce $010 x d 4000000000001510 \times 00 \times 00 \times 0 "$

\# Bus Unit (L2 Cache) Error (uncorrectable error)

mc2_inject $\quad=$ "mce $020 \times 66000000000201360 \times 0$ 0x0 0x0"

\# L1 Cache Data Store Error (uncorrectable error)

mc3_inject $\quad=$ "mce $030 \times 6002000000001450 \times 0$ 0x0 0x0"

\# ECC error (ADDR valid)

mc4_inject $\quad=$ "mce $040 \times 9426 \mathrm{c} 0010 \mathrm{~b} 0008130 \times 0 \quad 0 \times 0 \quad 0 \times 0 "$

mc4_inject_of $\quad=$ "mce $040 \times 1426 c 0010 b 0008130 \times 0 \quad 0 \times 0 \quad 0 \times 0 "$ 


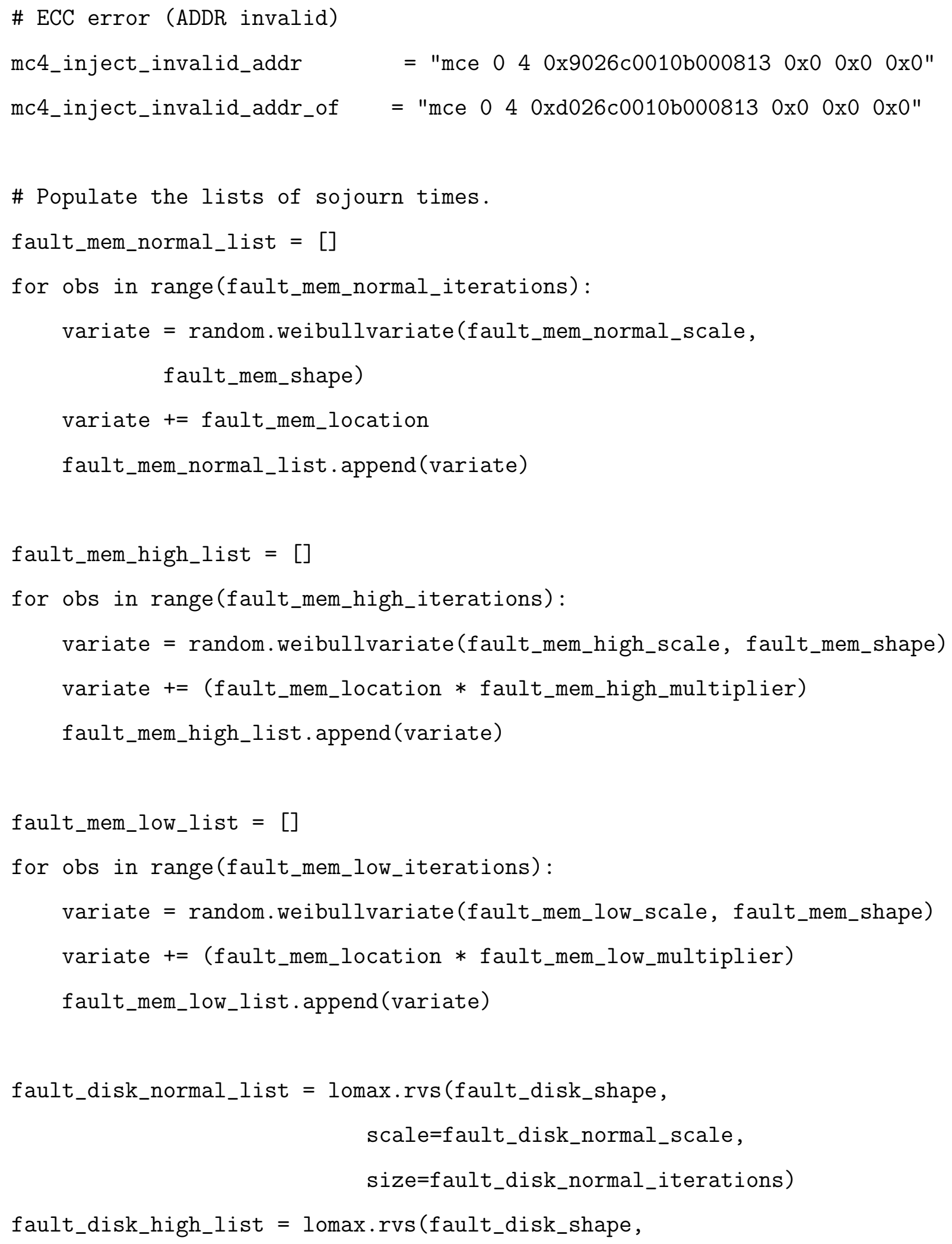




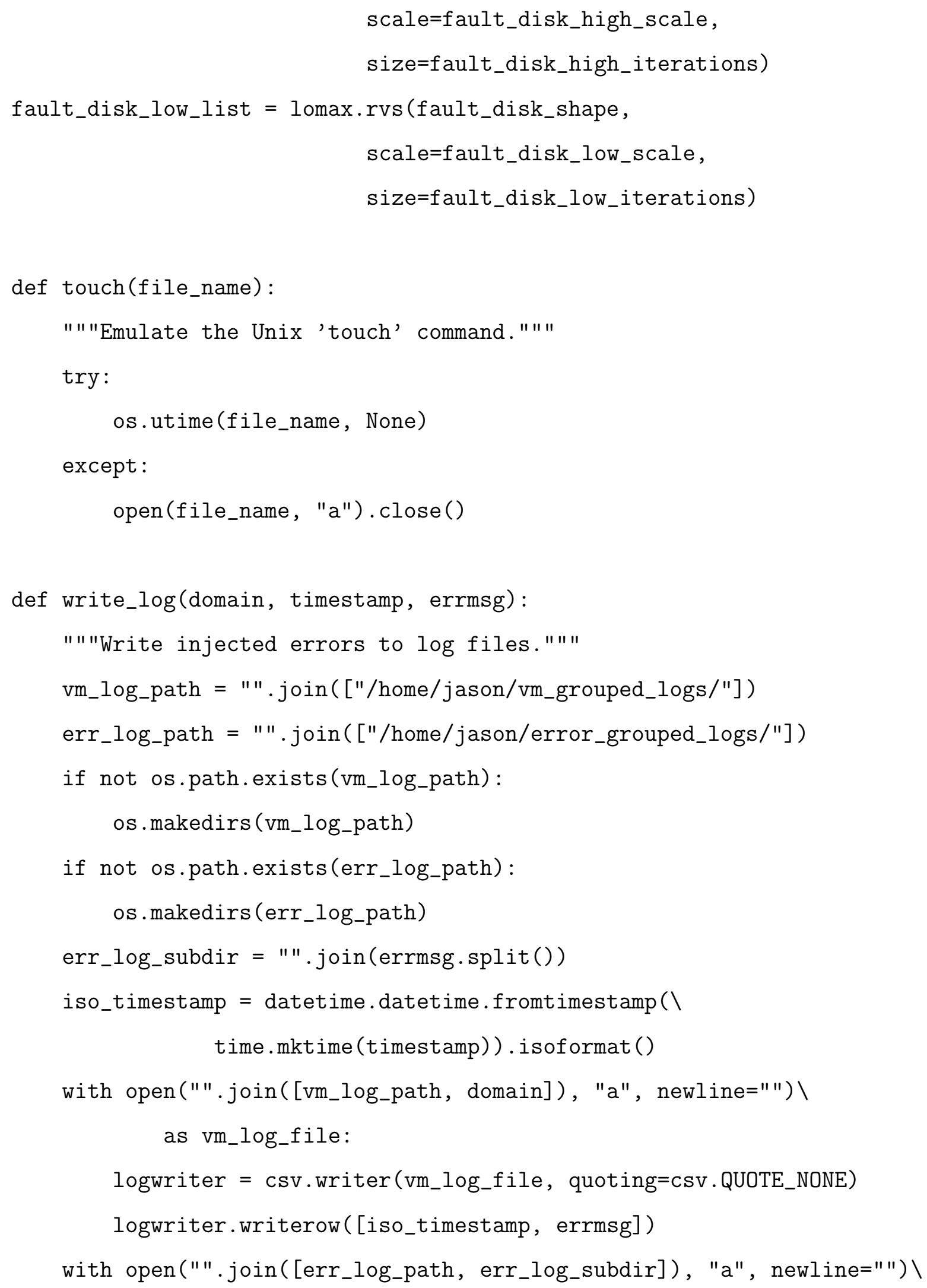


as errmsg_log_file:

logwriter = csv.writer(errmsg_log_file, quoting=csv.QUOTE_NONE) logwriter.writerow([iso_timestamp, domain])

def inject_error(domain, mac_addr, inject_string): "" Inject a fault into a virtual machine (domain).""

\# Handle disk errors separately from MCE errors if inject_string.startswith(("ENO", "EIN")): mac_addr_path = "".join([qemu_disk_inject_path, mac_addr, "/"]) file_path $=" "$ "join([mac_addr_path, inject_string $])$

if not os.path.exists(mac_addr_path): os.makedirs (mac_addr_path)

with open(file_path, "w"): touch (file_path)

time.sleep(0.01) \# let the file sit for a fraction of 1 second

\# remove the file, and hence, stop triggering the injection try:

os.remove(file_path)

except OSError as e:

if e.errno != errno.ENOENT: \# no such file or directory raise \# re-raise exception if a different error occurred

else:

try: subprocess.check_call(["virsh", "-c", "qemu:///system", 


$$
\begin{aligned}
& \text { "qemu-monitor-command " + domain + } \\
& \text { "--hmp --cmd \'" + inject_string + "\,"]) }
\end{aligned}
$$

except subprocess.CalledProcessError:

print("Error injecting [[", inject_string, "]] into domain", domain)

def injection_manager(domain_list, fault_list, intensity, inject_string): "" Manage the sojourn times and fault injections per fault type."" for seconds in fault_list:

\# Randomly select a target domain every injection step if intensity == 'high':

domain $=$ random. $\operatorname{choice}($ list $($ domain_list_high.keys ()$))$

mac_addr = domain_list_high[domain]

elif intensity $==$ 'normal':

domain $=$ random. $\operatorname{choice}($ list $($ domain_list_normal.keys ()$))$

mac_addr $=$ domain_list_normal[domain]

elif intensity $==$ 'low':

domain $=$ random. $\operatorname{choice}($ list $($ domain_list_low.keys ()$))$

mac_addr = domain_list_low [domain]

else:

print("Invalid intensity: ", intensity)

$\operatorname{exit}(3)$

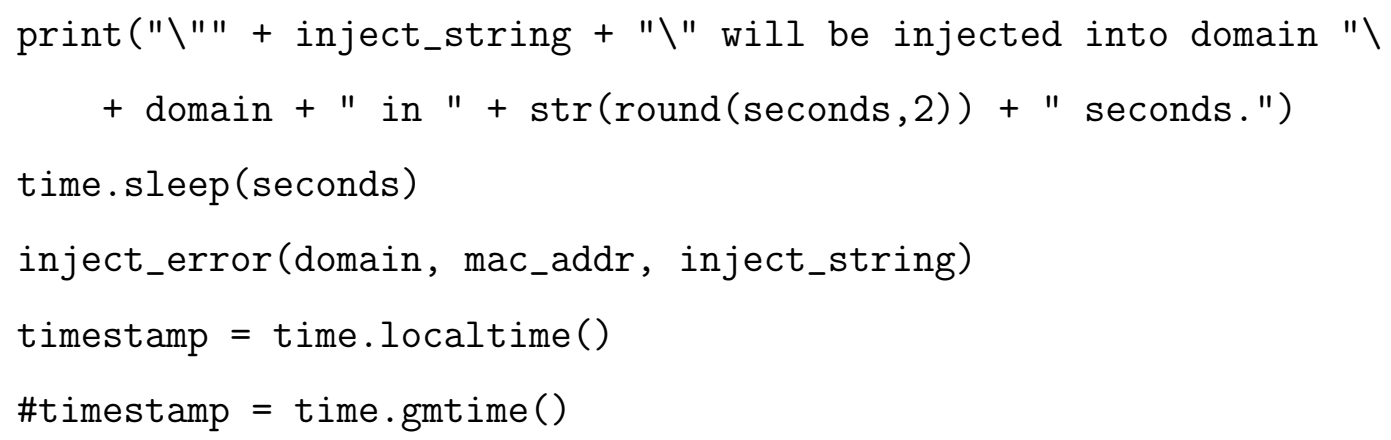


write_log(domain, timestamp, inject_string)

" I" "

Spawn a thread for each fault type. At each injection interval, each thread will randomly select a domain from the list of domains and inject one instance of its fault type. The thread will loop through the number of sojourn times.

" " " "

\# Memory errors

thread1 = threading. Thread (target=injection_manager, args $=($ domain_list_high, fault_mem_high_list, 'high', mc4_inject)) thread2 = threading. Thread(target=injection_manager, args $=($ domain_list_normal, fault_mem_normal_list, 'normal', mc0_inject)) thread3 = threading. Thread(target=injection_manager, args $=($ domain_list_low, fault_mem_low_list, 'low', mc1_inject)) \# Disk errors thread6 $=$ threading. Thread (target $=$ injection_manager, args $=($ domain_list_high, fault_disk_high_list, 'high', 'ENOMEDIUM')) thread7 = threading. Thread (target=injection_manager, args $=($ domain_list_normal, fault_disk_normal_list, 'normal', 'ENOMEM')) thread8 $=$ threading. Thread (target=injection_manager, args $=($ domain_list_low, fault_disk_low_list, 'low', 'EINVAL')) \# Threads must not be 'daemon threads' thread1. daemon $=$ False thread2 daemon $=$ False thread3. daemon $=$ False thread6 $\cdot$ daemon $=$ False thread 7 .daemon $=$ False thread8. daemon $=$ False 


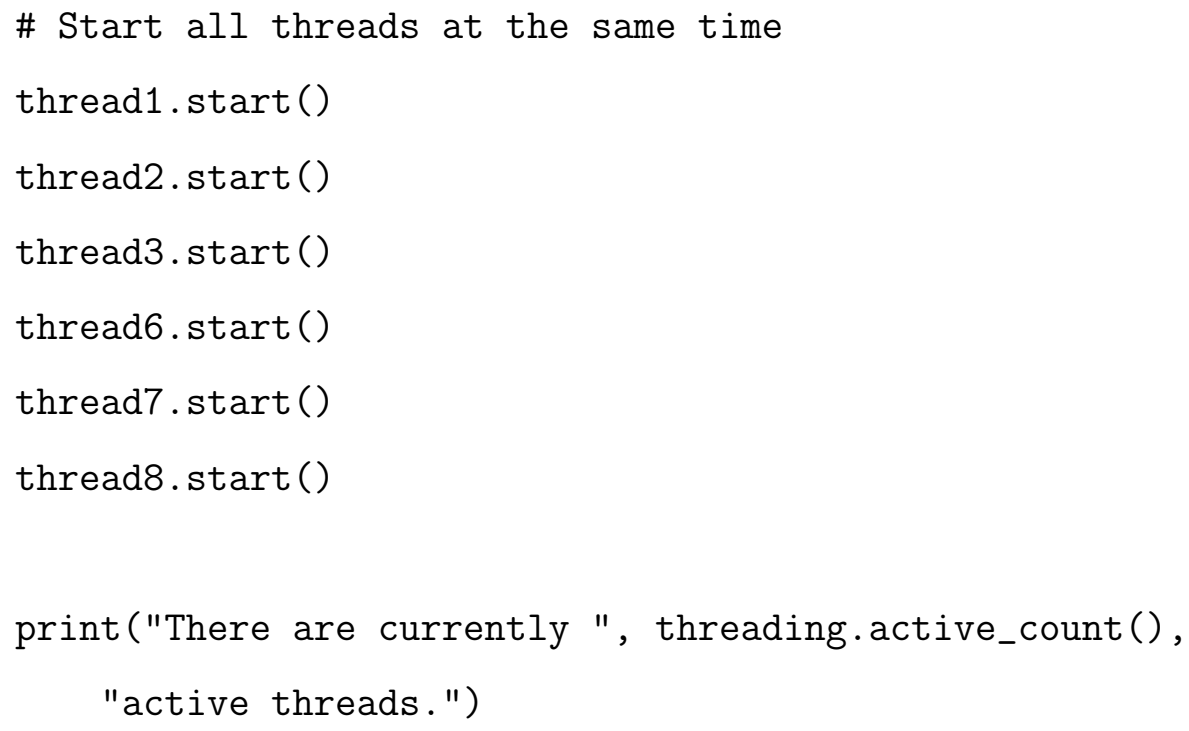


APPENDIX B. QEMU SOURCE CODE MODIFICATIONS

The next page begins the multi-page output of the "git diff" command that contains our modifications to the QEMU 2.0.0 source code. 
diff --git a/hw/scsi/scsi-disk.c b/hw/scsi/scsi-disk.c

index 48a28ae. .4359047 100644

--- a/hw/scsi/scsi-disk.c

+++ b/hw/scsi/scsi-disk.c

(@e $-37,10+37,14$ @@ do $\{\operatorname{printf}("$ scsi-disk: " fmt , \#\# _-VA_ARGS__); $\}$ while (0)

\#include "hw/block/block.h"

\#include "sysemu/dma.h"

+\#include "net/net.h"

$+$

\#ifdef ___linux

\#include <scsi/sg.h>

\#endif

+\#include <dirent.h>

\#define SCSI_WRITE_SAME_MAX 524288

\#define SCSI_DMA_BUF_SIZE 131072

\#define SCSI_MAX_INQUIRY_LEN 256

(e) $-292,6+296,159$ @e static void scsi_dma_complete(void *opaque, int ret) 
scsi_dma_complete_noio(opaque, ret);

\}

+/* Read a type of disk error to inject from the $\operatorname{dir} /$ tmp/qemu-disk-inject,

$+*$ and return it. The dir structure is

$+* /$ tmp/qemu-disk-inject/<MAC addr $>/<e r r$ type $>$

+ * When a VM matches the MAC address and has an empty file named one of

+ * "ENOMEDIUM", "ENOSPC", "EINVAL", or "ENOMEM", an error will be injected

$+*$ until the file is removed from the file system. */

+static int get_disk_error_to_inject(void)

$+\{$

$+\quad$ int err_to_inject $=0$;

$+\quad \mathrm{DIR} * \mathrm{dp}, * \mathrm{mdp}$

$+\quad$ struct dirent $*$ dresult, $*$ mdresult;

+ char macdir_name[1024];

+ int done $=0$;

$+\quad$ int macdone $=0$;

$+\quad$ macdone $=$ macdone +0 ;

$+\quad$ int $r c=0$;

$+\quad r c=r c+0$; 
+ char messages[128];

$+\quad$ strcpy(messages, "0");

$+\quad$ char vm_interface[100] = "net0";

$+\quad$ char $* v_{\text {vm_int; }}$

$+\quad$ int $i$

$+\quad$ int match;

+ uint8_t macaddr [6] ;

$+\quad$ int inaddr $[6]$;

$+\quad$ NetClientState $*$ ncs;

$+\quad$ NICState *nic_state;

$+$

$+\quad v_{-}$int $=v_{-}$interface;

$+\quad / /$ Find the running VM's MAC address

$+\quad$ ncs $=$ qemu_find_netdev_match(vm_int) ;

+ if (ncs $==$ NULL) \{

+ qemu_log("NetClientState pointer NULL for interface \%s $\backslash n$ ", vm_int);

$+\quad\}$ else \{

$+\quad$ nic_state $=$ qemu_get_nic(ncs); 
$+\quad$ if (nic_state $!=$ NULL) \{

$+\quad\}$

$+\quad\}$

$+\quad\}$

$+\quad$ errno $=0$;

$+\quad d p=$ opendir $(" / t m p / q e m u-d i s k-i n j e c t ") ;$

$+\quad$ if $(\mathrm{dp}==$ NULL $)\{$

+ $\quad$ perror("SCSI disk fault injection error: Main control config diropen failed");

$+\quad$ goto closefiles;

$+\quad\}$

$+\quad$ while (done $==0)\{$

+ // Iterate over MAC-named directories to find a match to current running VM

$+\quad$ dresult $=\operatorname{readdir}(\mathrm{dp})$; 
if $(($ dresult $==$ NULL $) \& \&($ errno $!=0))\{$

perror("SCSI disk fault injection error: Main control config readdir failed"); goto closefiles;

\}

if $(($ dresult $==$ NULL $) \& \&($ errno $==0))\{$

// No more directories.

done $=1$;

break;

\}

if (index(dresult->d_name, ' $\left.{ }^{\prime}\right) \quad !=$ NULL) \{

$r c=$ sscanf (dresult->d_name, $" \% \mathrm{x}: \% \mathrm{x}: \% \mathrm{x}: \% \mathrm{x}: \% \mathrm{x}: \% \mathrm{x} "$, \&inaddr[0], \&inaddr[1], \&inaddr [2], $\backslash$ \&inaddr [3], \&inaddr [4], \&inaddr [5]);

// Is this line intended for us?

$\operatorname{match}=1$;

for $(i=0 ; i<5 ; i++)\{$

if (inaddr $[i] !=\operatorname{macaddr}[i])\{$

match $=0$; 


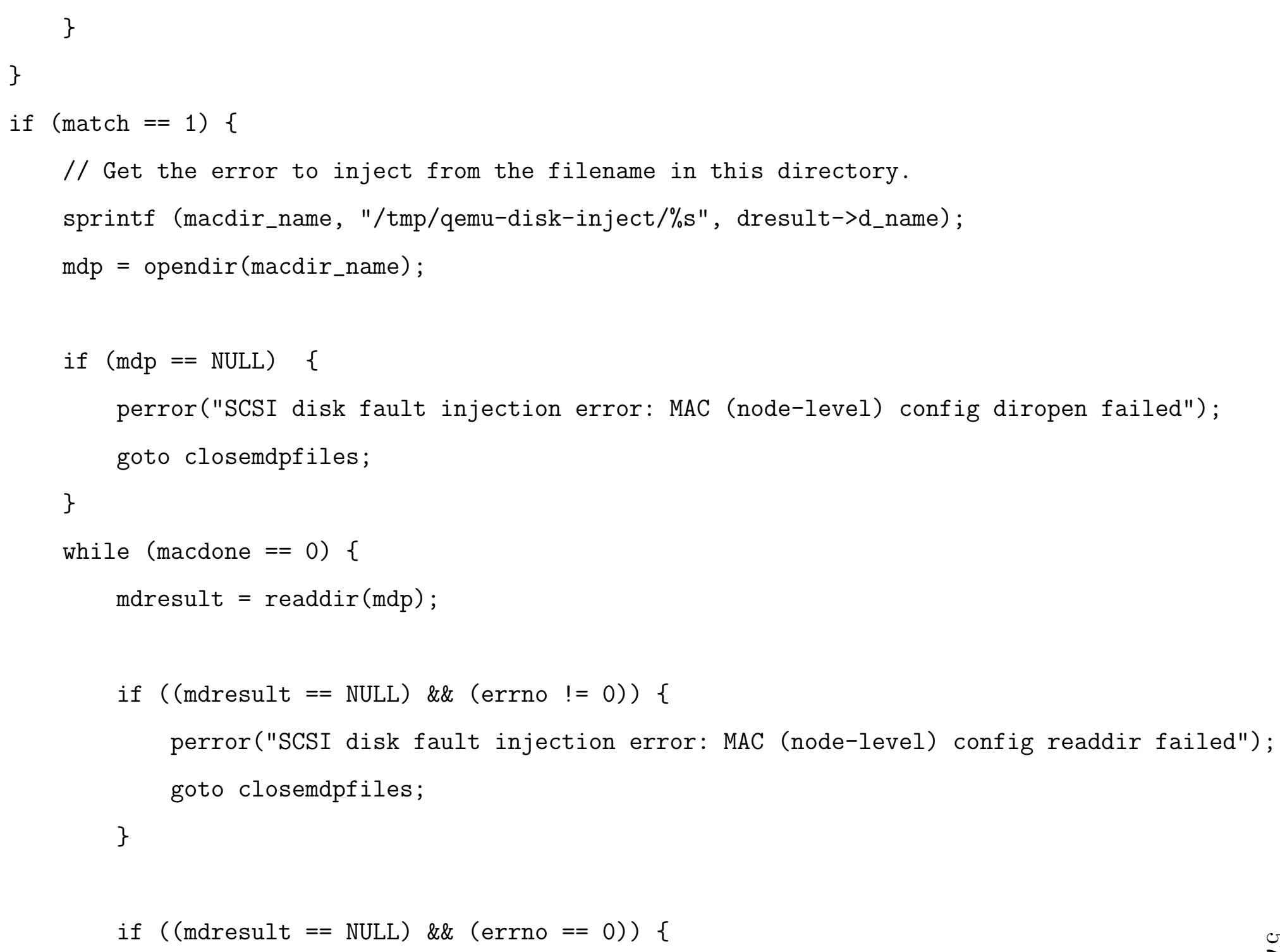


// Empty MAC directory

goto closemdpfiles;

\}

if (index(mdresult->d_name, '.') $==$ NULL) \{

// Scan the directory to discover the one message to be sent

if (strncmp (mdresult->d_name, "ENOMEDIUM", strlen ("ENOMEDIUM")) ==0) \{ err_to_inject $=$ ENOMEDIUM;

/* Remove injection support for ENOSPC because it suspends the VM immediately

* and the error isn't logged to syslog or the Journal.

$*$

* $\}$ else if (strncmp (mdresult->d_name, "ENOSPC", strlen("ENOSPC")) == 0) \{

* err_to_inject $=$ ENOSPC;

*/

\} else if (strncmp (mdresult->d_name, "EINVAL", strlen $(" E I N V A L "))=0$ ) \{ err_to_inject $=$ EINVAL;

\} else if (strncmp (mdresult->d_name, "ENOMEM", $\operatorname{strlen}(" E N O M E M "))=0$ ) \{ err_to_inject = ENOMEM;

\} else \{

err_to_inject $=0$;

\} 


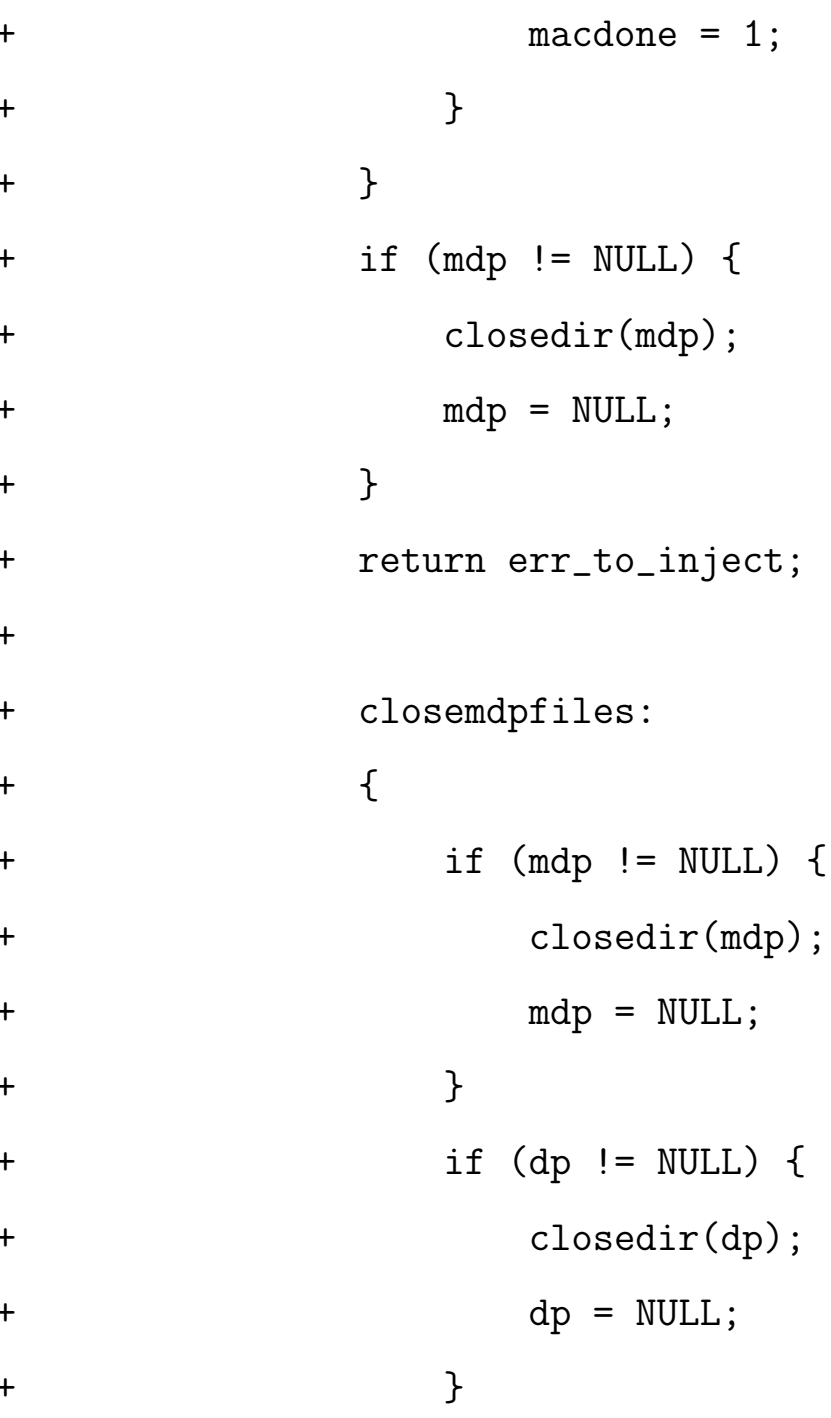




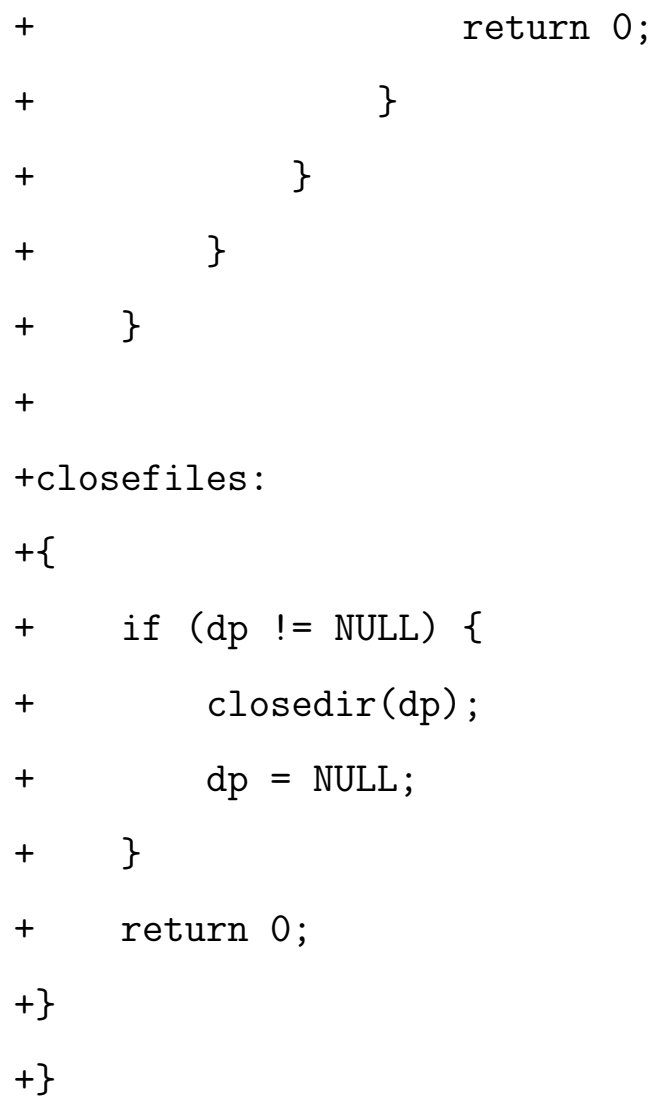


SCSIDiskReq $* r=$ opaque;

SCSIDiskState $* \mathrm{~s}=$ DO_UPCAST (SCSIDiskState, qdev, r->req.dev);

uint32_t $n$;

$+\quad$ int inject_err;

if ( $r$->req.aiocb != NULL) \{

$r->$ req.aiocb $=$ NULL;

(@) $-339,6+496,13$ @@ static void scsi_do_read(void *opaque, int ret) goto done;

\}

$+\quad$ inject_err $=$ get_disk_error_to_inject () ;

$+\quad$ if (inject_err) \{

$+\quad / /$ inject_err is either "0" or the value of a $C$ errno

$+\quad$ scsi_handle_rw_error( $r$, inject_err);

$+\quad$ return;

$+\quad\}$

$+$

if $(r e t<0)\{$

if (scsi_handle_rw_error $(r,-r e t))\{$ 
goto done;

(e) $-450,6+614,7$ @e static void scsi_write_complete(void $*$ opaque, int ret) SCSIDiskReq $* r=($ SCSIDiskReq $*)$ opaque;

SCSIDiskState $*$ S = DO_UPCAST (SCSIDiskState, qdev, r->req.dev); uint32_t $n$;

$+\quad$ int inject_err;

if $(r->$ req.aiocb $!=$ NULL $)\{$

$r->$ req. $a$ iocb $=$ NULL;

(e) $-459,6+624,13$ @e static void scsi_write_complete(void $*$ opaque, int ret) goto done;

\}

$+\quad$ inject_err $=$ get_disk_error_to_inject () ;

$+\quad$ if (inject_err) \{

$+\quad / /$ inject_err is either "O" or the value of a $C$ errno

$+\quad$ scsi_handle_rw_error(r, inject_err);

$+\quad$ return;

$+\quad\}$ 
if $(r e t<0)\{$

if (scsi_handle_rw_error $(r,-r e t))\{$

goto done;

diff --git a/include/net/net.h b/include/net/net.h

index 8166345.ffee769 100644

--- a/include/net/net.h

$+++\mathrm{b} /$ include/net/net.h

(@) $-98,6+98,7$ @e typedef struct NICState \{

bool peer_deleted;

\} NICState;

+NetClientState *qemu_find_netdev_match(const char *id);

NetClientState *qemu_find_netdev(const char $* i d)$;

int qemu_find_net_clients_except(const char $*$ id, NetClientState $* *$ ncs,

NetClientOptionsKind type, int $\max$ );

diff --git a/net/net.c b/net/net.c

index e3ef 1 e4..bbc6fd4 100644

--- a/net/net.c

$+++b /$ net/net.c

(e@ $-623,6+623,19$ @e NetClientState $*$ qemu_find_netdev(const char $*$ id) 
return NULL;

\}

+NetClientState *qemu_find_netdev_match(const char $*$ id)

$+\{$

$+\quad$ NetClientState $*$ nc;

$+$

+ QTAILQ_FOREACH(nc, \&net_clients, next) \{

$+\quad$ if $($ !strcmp (nc->name, id) $)\{$

$+\quad$ return nc;

$+\quad\}$

$+\quad\}$

$+$

$+\quad$ return NULL;

$+\}$

int qemu_find_net_clients_except(const char $*$ id, NetClientState $* *$ ncs, NetClientOptionsKind type, int $\max$ )

\{ 
LIST OF REFERENCES 


\section{LIST OF REFERENCES}

AMD64 architecture programmer's manual volume 2: System programming [Computer software manual]. (2011, May). Pub. 24593.

Atif, M., \& Strazdins, P. (2009). Optimizing live migration of virtual machines in SMP clusters for HPC applications. In Sixth IFIP international conference on network and parallel computing (pp. 51-58). Gold Coast, Queensland, AUS: IEEE. doi: 10.1109/NPC.2009.32

Axboe, J. (2015). fio: flexible I/O tester. Retrieved from https://github.com/axboe/fio

Bellard, F. (2014). QEMU: open-source processor emulator. Retrieved from http://qemu.org

BIOS and kernel developer's guide for AMD Athlon 64 and AMD Opteron processors [Computer software manual]. (2006, February). Pub. 26094.

DeBardeleben, N. A., Blanchard, S. P., Fu, S., Guan, Q., \& Zhang, Z. (2011). Experimental framework for injecting logic errors in a virtual machine to profile applications for soft error resilience (Tech. Rep.). Los Alamos, New Mexico, USA: Los Alamos National Laboratory. Retrieved from http://newmexicoconsortium.org/usrc/usrc-publications (LA-UR-11-10926)

EasyFit. (2010). MathWave Technologies. Retrieved from http://www . mathwave.com

Esteves, R. M., Hacker, T., \& Rong, C. (2012, December). Cluster analysis for the cloud: Parallel Competitive Fitness and parallel K-means++ for large dataset analysis. In Proceedings of the 2012 IEEE 4th international conference on cloud computing technology and science (pp. 177-184). Taipei, Taiwan: IEEE. doi: 10.1109/CloudCom.2012.6427553

Evangelinos, C., \& Hill, C. N. (2008). Cloud computing for parallel scientific HPC applications: Feasibility of running coupled atmosphere-ocean climate models on Amazon's EC2. In The first workshop on cloud computing and its applications '08. Retrieved from http://www.cca08.org/papers.html

Fu, S., \& Xu, C.-Z. (2007, October). Quantifying temporal and spatial correlation of failure events for proactive management. In Proceedings of the 26th IEEE international symposium on reliable distributed systems (pp. 175-184). Beijing, China: IEEE. doi: 10.1109/SRDS.2007.18 
Giuffrida, C., Kuijsten, A., \& Tanenbaum, A. S. (2013, December). EDFI: A dependable fault injection tool for dependability benchmarking experiments. In Proceedings of the 2013 IEEE 19th pacific rim international symposium on dependable computing (pp. 31-40). Vancouver, British Columbia, Canada: IEEE.

Guan, Q., DeBardeleben, N., Blanchard, S., \& Fu, S. (2014, May). F-SEFI: A fine-grained soft error fault injection tool for profiling application vulnerability. In Proceedings of the 2014 IEEE 28th international parallel and distributed processing symposium (pp. 1245-1254). Phoenix, Arizona: IEEE.

Hacker, T. J., Romero, R. F., \& Carothers, C. D. (2009). An analysis of clustered failures on large supercomputing systems. Journal of Parallel and Distributed Computing, 69(7), 652-665. doi: 10.1016/j.jpdc.2009.03.007

Kleen, A. (2015). mcelog: the Linux hardware error daemon. Retrieved from http://www.mcelog.org

Lange, J., Dinda, P., Xia, L., Bridges, P. G., Hale, K., et al. (2011). Palacios: An $O S$-independent embeddable VMM. Retrieved from

http: //www.v3vee.org/palacios/

Levy, S., Dosanjh, M. G. F., Bridges, P. G., \& Ferreira, K. B. (2013, June). Using unreliable virtual hardware to inject errors in extreme-scale systems. In Proceedings of the 3rd workshop on fault-tolerance for HPC at eXtreme scale (FTXS). New York, NY: ACM.

libvirt virtualization API. (2015). Red Hat, Inc. Retrieved from https://libvirt.org/

NIST. (2003, June). NIST/SEMATECH e-handbook of statistical methods. National Institute of Standards and Technology. Retrieved from

http://www.itl.nist.gov/div898/handbook/eda/section3/eda3668.htm

Oliner, A., \& Stearley, J. (2007, June). What supercomputers say: A study of five system logs. In Proceedings of the 37th annual IEEE/IFIP international conference on dependable systems $\&$ networks (pp. 575-584). Edinburgh, Scotland: IEEE. doi: 10.1109/DSN.2007.103

Pandit, N., Kalbarczyk, Z., \& Iyer, R. K. (2009, June / July). Effectiveness of machine checks for error diagnostics. In Proceedings of the IEEE/IFIP international conference on dependable systems 86 networks, 2009 (pp. 578-583). Lisbon, Portugal: IEEE. doi: 10.1109/DSN.2009.5270290

QEMU emulator user documentation: QEMU monitor. (n.d.). QEMU Project. Retrieved from http://wiki.qemu.org/download/qemu-doc.html

Romero, R. F. (2010). Live migration of parallel applications. Unpublished master's thesis, Purdue University, West Lafayette. Retrieved from http://docs.lib.purdue.edu/techmasters/27/ (UMI No. 1489538)

Rosen Center for Advanced Computing, P. U. (n.d.-a). ITaP research computing: Overview of Carter. Retrieved from https://www.rcac.purdue.edu/compute/carter/ 
Rosen Center for Advanced Computing, P. U. (n.d.-b). ITaP research computing: Overview of Coates. Retrieved from

https://www.rcac.purdue.edu/compute/coates/

The $R$ project for statistical computing. (2016). Retrieved from https://www.r-project.org

Salfner, F., \& Tschirpke, S. (2008, December). Error log processing for accurate failure prediction. In WASL'08 proceedings of the first USENIX conference on analysis of system logs (p. 4). San Diego, CA, USA: USENIX Association.

Schroeder, B., Damouras, S., \& Gill, P. (2010, February). Understanding latent sector errors and how to protect against them. In Proceedings of the 8th USENIX conference on file and storage technologies (pp. 71-84). San Jose, California: USENIX Association.

SciPy-Developers. (2013). SciPy: Scientific computing tools for Python. Retrieved from https://www.scipy.org

Thompson, D., Jiang, D., Peterson, D., Harbaugh, T., \& Chebab, M. C. (2011). EDAC - error detection and correction. Retrieved from https:// www . kernel.org/doc/Documentation/edac. txt

Torvalds, L., Red Hat, Inc., et al. (2010). The Red Hat Enterprise Linux 5 kernel source code. Retrieved from http://ftp.redhat.com/pub/redhat/linux/ enterprise/5Server/en/os/SRPMS/

Youseff, L., Wolski, R., Gorda, B., \& Krintz, C. (2006). Evaluating the performance impact of Xen on MPI and process execution for HPC systems. In Second international workshop on virtualization technology in distributed computing.

Zhang, Y., Squillante, M. S., Sivasubramaniam, A., \& Sahoo, R. K. (2004).

Performance implications of failures in large-scale cluster scheduling. Lecture Notes in Computer Science, 3277, 233-252. Retrieved from http://www.springer. com/computer/lncs

Zheng, Z., Lan, Z., Park, B. H., \& Geist, A. (2009). System log pre-processing to improve failure prediction. In Proceedings of the IEEE/IFIP international conference on dependable systems $\&$ networks, 2009 (pp. 572-577). Lisbon, Portugal: IEEE. doi: 10.1109/DSN.2009.5270289

Zhou, W., Zhan, J., Meng, D., Xu, D., \& Zhang, Z. (2010). LogMaster: Mining event correlations in logs of large-scale cluster systems. Computing Research Repository. Retrieved from http://arxiv.org/abs/1003.0951

Zhou, W., Zhan, J., Meng, D., \& Zhang, Z. (2010). Online event correlations analysis in system logs of large-scale cluster systems. Lecture Notes in Computer Science, 6289, 262-276. 